\title{
ON GLOBAL EXISTENCE FOR NONLINEAR WAVE EQUATIONS OUTSIDE OF CONVEX OBSTACLES
}

\author{
MARKUS KEEL, HART F. SMITH, AND CHRISTOPHER D. SOGGE
}

\section{Introduction.}

The purpose of this paper is to prove global existence for certain small-amplitude nonlinear Dirichlet-wave equations outside of smooth, strictly convex obstacles $\mathcal{K} \subset \mathbb{R}^{3}$. As in earlier works on the boundaryless case studied by Christodoulou [2] and Klainerman [10] we shall be concerned with equations where the nonlinearities involve a null form.

The null forms that we shall consider are the standard ones, which take the form

$$
Q_{0}(d v, d w)=\partial_{t} v \partial_{t} w-\sum_{j=1}^{3} \partial_{j} v \partial_{j} w
$$

or

$$
Q_{j k}(d v, d w)=\partial_{j} v \partial_{k} w-\partial_{k} v \partial_{j} w, \quad 0 \leq j<k \leq 3 .
$$

Here, $\partial_{j} v=\partial v / \partial x_{j}$ if $j=1,2,3$, and $\partial_{0} v=\partial_{t} v=\partial v / \partial t$. Recall that nonlinear hyperbolic systems that satisfy Klainerman's [9] null condition must involve linear combinations of these null forms. Furthermore, it is known (see [2] and [10]) that in Minkowski space there is global existence for small compactly supported data, while for other types of nonlinearities one can find arbitrarily small data with fixed compact support for which there is blowup. (See [8].)

Based on this we shall study nonlinear hyperbolic equations in the exterior of a strictly convex domain in $\mathbb{R}^{3}$ that satisfy the null condition. To be more specific, we shall fix a strictly convex obstacle $\mathcal{K} \subset \mathbb{R}^{3}$ with smooth boundary $\partial \mathcal{K}$.

We then shall consider nonlinear systems of the form

$$
\left\{\begin{array}{l}
\square u=Q(d u, d u), \quad(t, x) \in \mathbb{R}_{+} \times \mathbb{R}^{3} \backslash \mathcal{K} \\
\left.u(t, \cdot)\right|_{\partial \mathcal{K}}=0 \\
u(0, \cdot)=f, \partial_{t} u(0, \cdot)=g,
\end{array}\right.
$$

if $\square=\partial^{2} / \partial t^{2}-\Delta$ is the usual D'Alembertian. Here $u=\left(u^{1}, u^{2}, \ldots, u^{N}\right)$, and $Q=$ $\left(Q^{1}, \ldots, Q^{N}\right)$ with the $i$-th component of $Q$ being a linear combination of the null forms in (1.1) and (1.2). More specifically,

$$
Q^{i}=\sum_{j, k} a_{j, k}^{i} B_{j, k}^{i}\left(d u^{j}, d u^{k}\right),
$$

with $a_{j, k}^{i}$ being a constant and $B_{j, k}^{i}$ being any one of the null forms in (1.1)-(1.2).

The authors were supported in part by the NSF. 
We shall be interested in obtaining $H^{2}$ solutions of our equation. In view of the Dirichlet condition in (1.3) one must also then have that $\left.\partial_{t} u(t, \cdot)\right|_{\partial \mathcal{K}}=0$. As a consequence, one must assume that the data satisfy the compatibility conditions

$$
f(x)=0 \quad \text { and } \quad g(x)=0 \quad \text { if } x \in \partial \mathcal{K} .
$$

As in Christodoulou's [2] results for the non-obstacle case, we shall not have to assume that the data has compact support. Instead, this condition is replaced by a natural assumption that $f$ and $g$ belong to certain weighted Sobolev spaces.

To be more precise, let us first recall the weighted Sobolev spaces that were used by Christodoulou [2], which are given by the norm

$$
\|f\|_{H^{m, j}\left(\mathbb{R}^{3}\right)}=\sum_{|\alpha| \leq m}\left(\int_{\mathbb{R}^{3}}\left(1+|x|^{2}\right)^{|\alpha|+j}\left|D^{\alpha} f(x)\right|^{2} d x\right)^{1 / 2}
$$

Here we are using the standard notation $D^{\alpha}=\left(\partial / \partial x_{1}\right)^{\alpha_{1}}\left(\partial / \partial x_{2}\right)^{\alpha_{2}}\left(\partial / \partial x_{3}\right)^{\alpha_{3}}$, and $|\alpha|=$ $\alpha_{1}+\alpha_{2}+\alpha_{3}$. (Later in this paper $D^{\alpha}$ will involve time derivatives, but this will be clear from the context.) The associated weighted Dirichlet-Sobolev spaces for $m=1,2 \ldots$ then are

$$
H_{D}^{m, j}\left(\mathbb{R}^{3} \backslash \mathcal{K}\right)=\left\{f \in H^{m, j}\left(\mathbb{R}^{3} \backslash \mathcal{K}\right):\left.f\right|_{\partial \mathcal{K}}=0\right\}
$$

where $H^{m, j}\left(\mathbb{R}^{3} \backslash \mathcal{K}\right)$ is the space of restrictions of elements of $H^{m, j}\left(\mathbb{R}^{3}\right)$. Hence,

$$
\|f\|_{H_{D}^{m, j}}^{2}=\sum_{|\alpha| \leq m} \int_{\mathbb{R}^{3} \backslash \mathcal{K}}\left(1+|x|^{2}\right)^{|\alpha|+j}\left|D^{\alpha} f(x)\right|^{2} d x
$$

gives the natural norm on $H_{D}^{m, j}\left(\mathbb{R}^{3} \backslash \mathcal{K}\right)$. Also, $H^{m}\left(\mathbb{R}^{3} \backslash \mathcal{K}\right), m=1,2,3, \ldots$ will denote the Sobolev space of restrictions of elements of $H^{m}\left(\mathbb{R}^{3}\right)$, while $H_{D}^{m}\left(\mathbb{R}^{3} \backslash \mathcal{K}\right), m=1,2,3, \ldots$ will denote the subset $\left\{f \in H^{m}\left(\mathbb{R}^{3} \backslash \mathcal{K}\right):\left.f\right|_{\partial \mathcal{K}}=0\right\}$.

We can now state our first main result.

Theorem 1.1. Suppose that $f$ and $g$ satisfy (1.5) and that

$$
\|f\|_{H_{D}^{2,1}}+\|g\|_{H_{D}^{1,2}}<\varepsilon_{0} \text {. }
$$

Then if $\varepsilon_{0}>0$ is sufficiently small there is a unique global solution $u$ of (1.3) verifying

$$
\sup _{0<t<T} \sum_{|\alpha| \leq 2}\left\|D^{\alpha} u(t, \cdot)\right\|_{L^{2}\left(\mathbb{R}^{3} \backslash \mathcal{K}\right)}+\sum_{|\alpha| \leq 1}\left\|D^{\alpha} Q\right\|_{L^{2}\left([0, T] \times \mathbb{R}^{3} \backslash \mathcal{K}\right)}<\infty, \quad T>0 .
$$

Furthermore, the solution has the decay property

$$
|u(t, x)| \leq C(1+t)^{-1}
$$

Analogous results under the assumption of spherical symmetry for $\mathcal{K}$ and $u$ were obtained by Godin [4] if $Q=Q_{0}$. His proof involved an adaptation of Christodoulou's [2] method to this setting. If one drops the assumption of spherical symmetry, it does not seem that the arguments in [4] will apply in a straightforward way.

Previous work in higher dimensions applied Lorentz vector field techniques to the exterior problem. For general nonlinearities quadratic in $\nabla u, \partial_{t} u$, global smooth solutions were shown by Shibata and Tsutsumi [16] to exist in dimension $n \geq 6$. In Hayashi [6], 
global existence of smooth solutions outside of spheres in $n \geq 4$ is shown for a restricted class of quadratic nonlinearities, extending work in [5].

Our methods also give smooth solutions if the data satisfies the necessary compatibility conditions. To state these conditions, we define a collection of functions $\psi_{j}$ on $\mathbb{R}^{3} \backslash \mathcal{K}$ as follows. Set

$$
\psi_{0}=f, \quad \psi_{1}=g .
$$

We now define $\psi_{j}$ recursively so that, if the function $u_{c}$ has the following Taylor expansion in $t$,

$$
u_{c}(t, x) \approx \sum_{j=0}^{\infty} \psi_{j}(x) t^{j} / j !
$$

then $\square u_{c}-Q\left(d u_{c}, d u_{c}\right)$ vanishes to infinite order at $t=0$. This is seen to determine $\psi_{j}$ uniquely, and $\psi_{j}$ is a nonlinear function of the data $(f, g)$ that involves derivatives of order up to $j$ of $f$, and of order up to $j-1$ of $g$. If $(f, g) \in H^{k}\left(\mathbb{R}^{3} \backslash \mathcal{K}\right) \times H^{k-1}\left(\mathbb{R}^{3} \backslash \mathcal{K}\right)$ one would only use the first $k$ terms and require that the resulting function vanishes to order $k$ at $t=0$ if one wishes to obtain $H^{k}$ solutions.

Definition 1.2. We say that the data $(f, g)$ satisfies the compatibility conditions to order $k$ if, for $0 \leq j \leq k$, the functions $\psi_{j}$ vanish on $\partial \mathcal{K}$. We say that the data satisfies the compatibility conditions to infinite order if all the $\psi_{j}$ vanish on $\partial \mathcal{K}$.

Note that the assumption in Theorem 1.1 was that the data satisfies the first order compatibility condition. If $\bar{f}$ and $\bar{g}$ are appropriate extensions of $f$ and $g$, respectively, to all of $\mathbb{R}^{3}$ and if $\bar{u}$ is a local solution to the equation $\square \bar{u}=Q(d \bar{u}, d \bar{u})$ one can just take $\psi_{j}(x)=\partial_{t}^{j} \bar{u}(0, x), x \in \mathbb{R}^{3} \backslash \mathcal{K}$. Then the $k$ th order compatibility condition would be equivalent to the condition that $\partial_{t}^{j} \bar{u}(0, x)=0$, when $0 \leq j \leq k$ and $x \in \partial \mathcal{K}$. Also note that all of the compatibility conditions are automatically satisfied if the data vanishes near $\partial \mathcal{K}$.

Theorem 1.3. Assume that $(f, g) \in C^{\infty}\left(\mathbb{R}^{3} \backslash \mathcal{K}\right)$ satisfies the compatibility conditions to infinite order and that

$$
\|f\|_{H_{D}^{2,1}}+\|g\|_{H_{D}^{1,2}}<\varepsilon_{0} .
$$

Then if $\varepsilon_{0}>0$ is sufficiently small there is a unique solution $u \in C^{\infty}\left(\mathbb{R}_{+} \times \mathbb{R}^{3} \backslash \mathcal{K}\right)$ of (1.3). This solution also satisfies (1.10).

Our approach will be to combine the conformal method introduced in [1] (see also [2]) with techniques developed in [3], [11], [17] and [20]. These results all involve generalizations of an inequality of Klainerman and Machedon 11. In the scalar case, it involves estimates for solutions $u$ of linear wave equations in Minkowski space $\mathbb{R}_{+}^{1+3}$

$$
\left\{\begin{array}{l}
\square u=F, \quad(t, x) \in \mathbb{R}_{+}^{1+3} \\
u(t, \cdot)=u_{0}, \quad \partial_{t} u(t, \cdot)=u_{1} .
\end{array}\right.
$$


To be more specific, if $v$ solves the same equation with data $\left(v_{0}, v_{1}, G\right)$, then the main estimate in [11] says that if $Q$ is any of the null forms in (1.1)-(1.2) then

$$
\begin{aligned}
\sum_{|\alpha| \leq 1}\left\|D^{\alpha} Q(d u, d v)\right\|_{L^{2}\left(\mathbb{R}_{+}^{1+3}\right)} \leq C( & \left.\left\|u_{0}\right\|_{H^{2}}+\left\|u_{1}\right\|_{H^{1}}+\int_{0}^{\infty}\|F(t, \cdot)\|_{H^{1}} d t\right) \\
& \times\left(\left\|v_{0}\right\|_{H^{2}}+\left\|v_{1}\right\|_{H^{1}}+\int_{0}^{\infty}\|G(t, \cdot)\|_{H^{1}} d t\right) .
\end{aligned}
$$

This estimate was extended locally to the boundaryless manifold case by the last author in 20]. Since we shall use a variant of Christodoulou's conformal method, this will be one of the ingredients in the proof of our existence results. In particular, the results from [20] in the special case where the underlying spatial manifold is $S^{3}$ will allow us to prove the necessary estimates when we are away from the boundary in the image of $\mathbb{R}_{+} \times \mathbb{R}^{3} \backslash \mathcal{K}$ via Penrose's [15] conformal compactification.

Proving estimates near the boundary in the image of $\mathbb{R}_{+} \times \mathbb{R}^{3} \backslash \mathcal{K}$, though, is much more complicated. This is because of the fact that the fixed-time cross sections of this set are hypersurfaces that vary with time in $[0, \pi) \times S^{3}$, and, in fact, degenerate to a point as the time variable $T$ tends to $\pi$.

To get around this it turns out to be convenient to pull back our estimates for the image of $\mathbb{R}_{+} \times \mathbb{R}_{+}^{3} \backslash \mathcal{K}$ to Minkowski space. Here we shall be able to prove the resulting estimates by applying energy decay estimates of Lax, Morawetz and Phillips [12]-13] along with local estimates for the inhomogeneous Dirichlet-wave equation. The latter were recently obtained by the last two authors in 18 .

Let us be more specific.

The classical energy estimates from [12]-13] that we shall require are contained in the following

Theorem 1.4. (Lax-Morawetz-Phillips [12]-113]) Suppose that u solves the homogeneous Dirichlet-wave equation $\square u=0$ in $\mathbb{R}_{+} \times \mathbb{R}^{3} \backslash \mathcal{K},\left.u\right|_{\mathbb{R}_{+} \times \partial \mathcal{K}}=0$. Suppose further that the Cauchy data $u_{0}=\left.u\right|_{t=0}$ and $u_{1}=\left.\partial_{t} u\right|_{t=0}$ vanish when $|x|>A$, with A fixed. Then there are constants $c>0$ and $C<+\infty$, depending on $\mathcal{K}$ and $A$, so that if $D u=\left(\partial_{t} u, \nabla_{x} u\right)$

$$
\sum_{|\alpha| \leq 1} \int_{\left\{x \in \mathbb{R}^{3} \backslash \mathcal{K}:|x|<A\right\}}\left|D^{\alpha} u(t, x)\right|^{2} d x \leq C e^{-c t} \sum_{|\alpha| \leq 1} \int_{\left\{x \in \mathbb{R}^{3} \backslash \mathcal{K}:|x|<A\right\}}\left|D^{\alpha} u(0, x)\right|^{2} d x .
$$

We shall actually require a consequence of (1.14) that involves estimates for higher derivatives. These follow from (1.14) and elliptic regularity arguments (cf. 116). If we use Duhamel's formula we can also get estimates for the inhomogeneous wave equation

$$
\left\{\begin{array}{l}
\square u(t, x)=F(t, x), \quad(t, x) \in \mathbb{R}_{+} \times \mathbb{R}^{3} \backslash \mathcal{K} \\
u(t, x)=0, \quad x \in \partial \mathcal{K} \\
u(0, x)=u_{0}(x), \quad \partial_{t} u(0, x)=u_{1}(x)
\end{array}\right.
$$


Specifically, we first notice that (1.14) yields

$$
\begin{aligned}
\sum_{|\alpha| \leq 2}\left\|D^{\alpha} u(t, \cdot)\right\|_{L^{2}\left(\left\{x \in \mathbb{R}^{3} \backslash \mathcal{K}:|x|<A\right\}\right)} & \\
& \leq C e^{-c t}\left[\left\|u_{0}\right\|_{H_{D}^{2}}+\left\|u_{1}\right\|_{H_{D}^{1}}+\sum_{|\alpha| \leq 1}\left\|e^{c s} D^{\alpha} F\right\|_{L_{t}^{1} L_{x}^{2}\left([0, t] \times \mathbb{R}^{3} \backslash \mathcal{K}\right)}\right],
\end{aligned}
$$

for constants $c>0$ and $C<+\infty$ as above provided that $u_{j}(x)=0, j=0,1$, and $F(t, x)=0$ when $|x|>A$. Note that if we use the Schwarz inequality then we can dominate the last term by

$$
C \sum_{|\alpha| \leq 1}\left\|e^{-c(t-s) / 2} D^{\alpha} F\right\|_{L^{2}\left([0, t] \times \mathbb{R}^{3} \backslash \mathcal{K}\right)} .
$$

If we combine the last two inequalities we obtain the following useful result.

Proposition 1.5. Fix $A$ and suppose that $u$ solves (1.15) with Cauchy data $u_{j}(x), j=$ 0,1 , and forcing term $F(t, x)$ both vanishing when $|x|>A$. Then there are constants $c>0$ and $C<+\infty$ depending only on $A$ and $\mathcal{K}$ so that

$$
\begin{aligned}
& \sum_{|\alpha| \leq 2}\left\|D^{\alpha} u(t, \cdot)\right\|_{L^{2}\left(\left\{x \in \mathbb{R}^{3} \backslash \mathcal{K}:|x|<A\right\}\right)} \\
& \quad \leq C e^{-c t}\left(\left\|u_{0}\right\|_{H_{D}^{2}}+\left\|u_{1}\right\|_{H_{D}^{1}}\right)+C \sum_{|\alpha| \leq 1}\left\|e^{-c(t-s)} D^{\alpha} F\right\|_{L^{2}\left([0, t] \times \mathbb{R}^{3} \backslash \mathcal{K}\right)} .
\end{aligned}
$$

The local null form estimates that we shall need are:

Theorem 1.6. (Smith-Sogge [18]) Suppose that $u$ and $v$ satisfy inhomogeneous Dirichletwave equations $\square u=F$ and $\square v=G$ in $\mathbb{R}_{+} \times \mathbb{R}^{3} \backslash \mathcal{K}$ with Cauchy data $\left(u_{0}, u_{1}\right)$ and $\left(v_{0}, v_{1}\right)$, respectively. Then if $Q$ is any of the null forms in (1.1)-(1.2)

$$
\begin{aligned}
& \sum_{|\alpha| \leq 1}\left\|D^{\alpha} Q(d u, d v)\right\|_{L^{2}\left([0,1] \times \mathbb{R}^{3} \backslash \mathcal{K}\right)} \\
& \leq C\left(\left\|u_{0}\right\|_{H_{D}^{2}}+\left\|u_{1}\right\|_{H_{D}^{1}}+\sum_{|\alpha| \leq 1}\left\|D^{\alpha} F(t, \cdot)\right\|_{L^{1} L^{2}\left([0,1] \times \mathbb{R}^{3} \backslash \mathcal{K}\right)}\right) \\
& \quad \times\left(\left\|v_{0}\right\|_{H_{D}^{2}}+\left\|v_{1}\right\|_{H_{D}^{1}}+\sum_{|\alpha| \leq 1}\left\|D^{\alpha} G(t, \cdot)\right\|_{L^{1} L^{2}\left([0,1] \times \mathbb{R}^{3} \backslash \mathcal{K}\right)}\right) .
\end{aligned}
$$

This equation of course gives small $H^{2}$ data local existence for (1.3) (see 118.) Unfortunately, though, it does not yield global existence. To get around this, we shall prove a weighted variant in the image of $\mathbb{R}_{+} \times \mathbb{R}^{3} \backslash \mathcal{K}$ in the Einstein diamond. (This estimate would in turn pull back to a global weighted variant of (1.17), but we do not explore that in this paper.) This paper is organized as follows. In the next section we shall review Penrose's [15] conformal compactification of Minkowski space to the so called Einstein diamond in $(-\pi, \pi) \times S^{3}$. We shall collect the necessary facts regarding the way that our nonlinear equation (1.3) transforms and state our main estimate in $[0, \pi) \times S^{3}$ that leads to global existence. It will be a weighted analog of $(1.17)$ on $[0, \pi) \times S^{3}$ minus the image of $\mathbb{R}_{+} \times \mathcal{K}$. The weights will compensate for the degeneracies of the boundary as $T \rightarrow \pi$. In subsequent sections we shall prove our main estimate using the strategy mentioned above of proving things directly outside of an appropriate neighborhood of the boundary, 
while proving things near the boundary by pulling everything back to Minkowski space. Finally, after we prove the weighted estimates we shall see how our estimates give the $H^{2}$ and $C^{\infty}$ global existence theorems.

\section{Conformal Compactification and the Main Estimate.}

Consider polar coordinates on the sphere $S^{3}$ minus the south pole given by

$$
[0, \pi) \times S^{2} \ni(R, \omega) \rightarrow(\cos R, \omega \sin R)=X=\left(X_{0}, \vec{X}\right) \in \mathbb{R}^{4} .
$$

Then the "standard" Lorentz metric on $\mathbb{R} \times S^{3}$ is given by

$$
g=d X^{2}=d T^{2}-d R^{2}-\sin ^{2} R d \omega^{2} .
$$

Since we are interested in solutions of (1.3) it is natural to consider another metric coming from Penrose's conformal compactification of $\mathbb{R}^{1+3}=\mathbb{R} \times \mathbb{R}^{3}$. This "physical metric" is just the pushforward of the standard Lorentz metric in Minkowski space, and it turns out to be conformally equivalent to (2.2). The facts we shall state about this transformation are well known and can be found, for example, in Hörmander [7].

Let us be more specific. First of all, we define the Einstein cylinder to be the set

$$
\mathbb{E}^{1+3}=(-\pi, \pi) \times S^{3} .
$$

We also need to define the Einstein diamond

$$
\overline{\mathbb{E}}^{1+3} \subset \mathbb{E}^{1+3}
$$

which is just the proper subset of $(-\pi, \pi) \times S^{3}$ given by

$$
\overline{\mathbb{E}}^{1+3}=\{(T, \cos R, \omega \sin R):-\pi<T<\pi, 0 \leq R<\pi, R+|T|<\pi\} .
$$

Penrose's transformation in [15] then is the conformal map $\mathcal{P}: \mathbb{R}^{1+3} \rightarrow \overline{\mathbb{E}}^{1+3}$, which in polar coordinates is defined by

$$
\begin{aligned}
(t, r, \omega) \rightarrow & (T, R, \omega), \\
& T=\tan ^{-1}(t+r)+\tan ^{-1}(t-r), R=\tan ^{-1}(t+r)-\tan ^{-1}(t-r),
\end{aligned}
$$

where as usual $x=r \omega, r=|x|$ in $\mathbb{R}^{3}$. Note that the inverse of $\mathcal{P}$ is given by

$$
\mathcal{P}^{-1}: \overline{\mathbb{E}}^{1+3} \ni(T, X) \rightarrow \frac{1}{\cos T+X_{0}}(\sin T, \vec{X}) \in \mathbb{R}^{1+3} .
$$

Notice also that when $T=0$ the map (2.5) is stereographic projection of $S^{3}$ from the south pole $(-1,0,0,0)$.

Under this map the pushforward of the Minkowski metric $d t^{2}-d x^{2}$ is the Lorentz metric $\tilde{g}$ in $\overline{\mathbb{E}}^{1+3}$ defined by

$$
g=\Omega^{2} \tilde{g},
$$

where the conformal factor is given by

$$
\Omega=\cos T+\cos R=\frac{2}{\left(1+(t+r)^{2}\right)^{1 / 2}\left(1+(t-r)^{2}\right)^{1 / 2}},
$$

with $(t, r)$ and $(T, R)$ being identified as in $(2.4)$. 
Continuing, let

$$
\square_{g}=\partial^{2} / \partial T^{2}-\Delta_{S^{3}}
$$

be the D'Alembertian coming from the standard Laplace-Beltrami operator, $\Delta_{S^{3}}$, given by the round metric $d T^{2}-d X^{2}$. On the other hand, $\tilde{\square}$ will be the D'Alembertian on $\mathbb{R}^{1+3}$ or $\overline{\mathbb{E}}^{1+3}$, depending on the context, that arises from $d t^{2}-d x^{2}$. In Minkowski space it is of course the standard D'Alembertian $\square=\partial^{2} / \partial t^{2}-\Delta$. A key fact for us will be the way the two D'Alembertians are related in $\overline{\mathbb{E}}^{1+3}$ :

$$
\square_{g}+1=\Omega^{-3} \tilde{\square} \Omega,
$$

with the additive constant 1 arising from the non-zero scalar curvature of $g$. Equivalently,

$$
\left(\square_{g}+1\right) u=F \Longleftrightarrow \tilde{\square} \tilde{u}=\tilde{F} \text { with } \tilde{u}=\Omega u \text { and } \tilde{F}=\Omega^{3} F .
$$

Another related standard fact concerns the way that the Sobolev spaces $(1.6)$ transform. For this we need to let $\mathcal{P}_{0}(\mathcal{K})$ denote the image of $\mathcal{K}$ under the restriction of the Penrose transformation to $\left\{(0, x): x \in \mathbb{R}^{3}\right\}$. Since $\mathcal{P}_{0}$ is the stereographic projection map, $\mathcal{P}_{0}(\mathcal{K}) \subset S^{3}$ is a convex set with smooth boundary. For $m=1,2, \ldots$ we then let $H_{D}^{m}\left(S^{3} \backslash \mathcal{P}_{0}(\mathcal{K})\right)=\left\{f \in H^{m}\left(S^{3} \backslash \mathcal{P}_{0}(\mathcal{K})\right):\left.f\right|_{\partial \mathcal{P}_{0}(\mathcal{K})}=0\right\}$ be the Sobolev space defined as before. If then $\mathcal{P}_{0}^{*} h$ denotes the pullback of a function $h$ on $S^{3}$ via the above restriction of the Penrose transformation it follows that the mapping

$$
\tilde{f}=\Omega \mathcal{P}_{0}^{*} f \rightarrow f
$$

is a continuous map from $H^{m, m-1}\left(\mathbb{R}^{3} \backslash \mathcal{K}\right)$ to $H^{m}\left(S^{3} \backslash \mathcal{P}_{0}(\mathcal{K})\right)$. That is, for every $m=$ $1,2,3, \ldots$ there is a constant $C_{m}<\infty$ so that

$$
\|f\|_{H^{m}\left(S^{3} \backslash \mathcal{P}_{0}(\mathcal{K})\right)} \leq C_{m}\left\|\Omega \mathcal{P}_{0}^{*} f\right\|_{H^{m, m-1}\left(\mathbb{R}^{3} \backslash \mathcal{K}\right)}=C_{m}\|\tilde{f}\|_{H^{m, m-1}\left(\mathbb{R}^{3} \backslash \mathcal{K}\right)} .
$$

Notice that if $u$ and $\tilde{u}$ are related as above then this inequality yields

$$
\left\|\partial_{T} u(0, \cdot)\right\|_{H^{m-1}\left(S^{3} \backslash \mathcal{P}_{0}(\mathcal{K})\right)} \leq C_{m}\left\|\partial_{t} \tilde{u}(0, \cdot)\right\|_{H^{m-1, m}\left(\mathbb{R}^{3} \backslash \mathcal{K}\right)},
$$

since the pushforward of $\partial / \partial t$ is $\Omega \partial / \partial T$ when $t=0$ and since $\Omega=2 /\left(1+|x|^{2}\right)$ when $t=0$.

Another fact that we shall use concerns the image of $\mathbb{R}_{+} \times \mathcal{K}$ in $\overline{\mathbb{E}}_{+}^{1+3}=\overline{\mathbb{E}}^{1+3} \cap[0, \pi) \times S^{3}$. Let us call this set

$$
\mathcal{K}_{*}=\mathcal{P}\left(\mathbb{R}_{+} \times \mathcal{K}\right) .
$$

Then one can check using (2.5) that there is a uniform constant $0<C<\infty$ so that for $0 \leq T<\pi$

$$
C^{-1}(\pi-T)^{2} \leq \operatorname{dist}(X, \mathbf{1}) \leq C(\pi-T)^{2} \quad \text { if } \quad(T, X) \in \partial \mathcal{K}_{*} .
$$

Here

$$
\mathbf{1}=(1,0,0,0)
$$

is the north pole and $\operatorname{dist}(\cdot, \cdot)$ is the standard distance on $S^{3}$ (induced by the metric $g$ ). If we let

$$
P_{0}=(\pi, \mathbf{1})
$$


be the convergence of the positive time-like infinities then we can state (2.12) in an equivalent way by saying that

$$
C^{-1} \operatorname{dist}^{2}\left(P, P_{0}\right) \leq \operatorname{dist}(X, \mathbf{1}) \leq C \operatorname{dist}^{2}\left(P, P_{0}\right), \text { if } P=(T, X) \in \partial \mathcal{K}_{*} .
$$

It should be clear from the context that we are using dist $(\cdot, \cdot)$ in two different ways. The distance between two points on $S^{3}$ is the distance given by the restriction of $g$ to $S^{3}$ and the distance between two points on $\mathbb{R} \times S^{3}$ is given by $g$. We shall use this notation in what follows.

The fact that the boundary of $\mathcal{K} *$ varies with time and, moreover, degenerates to a point as $T \rightarrow \pi$ is the reason that Christodoulou's [2] approach of using the above conformal compactification and the energy integral method does not seem to apply in an easy way for (1.3). In particular, as we shall see, it does not seem easy to control fixedtime high order Sobolev norms of a solution of the Dirichlet-wave equation in $\overline{\mathbb{E}}_{+}^{1+3} \backslash \mathcal{K}_{*}$ as $T \rightarrow \pi$, unless one is willing to include weights in the norms involving powers of $\operatorname{dist}^{2}\left(P, P_{0}\right)$.

We are almost ready to state our main inequality. To motivate the weights we shall use, let us first recall how the standard vector fields in Minkowski space pushforward to ones in $\overline{\mathbb{E}}^{1+3}$ via $\mathcal{P}$. We shall follow the exposition in Hörmander [7], p. 277-282.

To do this it is convenient to use stereographic projection coordinates on $S^{3}$. As we pointed out before the south pole stereographic projection coordinates come from the restriction of $\mathcal{P}^{-1}$ to $T=0$ :

$$
Y=\mathcal{P}_{0}^{-1}(X)=\frac{\sin R}{1+\cos R} \omega=\tan \left(\frac{R}{2}\right) \omega .
$$

The coordinates coming from the stereographic north pole projection arise from these and the Kelvin transform

$$
Z_{j}=Y_{j} /|Y|^{2}
$$

To compute the pushforwards of vector fields on $\overline{\mathbb{E}}^{1+3}$ it is convenient to use the vector fields

$$
\frac{\partial}{\partial T}, X_{j} \frac{\partial}{\partial X_{k}}-X_{k} \frac{\partial}{\partial X_{j}}, 0 \leq j<k \leq 3 .
$$

For future reference, let us arrange these as $\Gamma=\left\{\Gamma_{0}, \ldots, \Gamma_{6}\right\}$ and write $\Gamma^{\alpha}=\Gamma_{0}^{\alpha_{0}} \cdots \Gamma_{6}^{\alpha_{6}}$. Note that each $\Gamma$ is actually a smooth vector field on $\mathbb{E}^{1+3}$.

With this notation we can state the following result (see [7])

Proposition 2.1. The pushforwards of $\partial / \partial t$ and $\partial / \partial x_{j}, 1 \leq j \leq 3$ in $\mathbb{R}^{1+3}$ by $\mathcal{P}$ are

$$
\begin{aligned}
\frac{\partial}{\partial t} & =\left(1+\frac{1-|Y|^{2}}{1+|Y|^{2}} \cos T\right) \frac{\partial}{\partial T}-\sin T\left\langle Y, \frac{\partial}{\partial Y}\right\rangle \\
& =\left(1+\frac{|Z|^{2}-1}{|Z|^{2}+1} \cos T\right) \frac{\partial}{\partial T}+\sin T\left\langle Z, \frac{\partial}{\partial Z}\right\rangle,
\end{aligned}
$$


and

$$
\begin{aligned}
\frac{\partial}{\partial x_{j}} & =\frac{-2 Y_{j}}{1+|Y|^{2}} \sin T \frac{\partial}{\partial T}+\frac{1}{2}\left(\left(1+|Y|^{2}\right) \cos T+1-|Y|^{2}\right) \frac{\partial}{\partial Y_{j}}+(1-\cos T) Y_{j}\left\langle Y, \frac{\partial}{\partial Y}\right\rangle \\
& =\frac{-2 Z_{j}}{1+|Z|^{2}} \sin T \frac{\partial}{\partial T}+\frac{1}{2}\left(\left(1+|Z|^{2}\right) \cos T+|Z|^{2}-1\right) \frac{\partial}{\partial Z_{j}}+(1+\cos T) Z_{j}\left\langle Z, \frac{\partial}{\partial Z}\right\rangle .
\end{aligned}
$$

The pushforward of the vector fields in (2.17) via $\mathcal{P}^{-1}$ are given by

$$
\begin{aligned}
& X_{j} \frac{\partial}{\partial X_{k}}-X_{k} \frac{\partial}{\partial X_{j}}=x_{j} \frac{\partial}{\partial x_{k}}-x_{k} \frac{\partial}{\partial x_{j}}, 1 \leq j<k \leq 3, \\
& X_{0} \frac{\partial}{\partial X_{k}}-X_{k} \frac{\partial}{\partial X_{0}}=\frac{1}{2}\left(1+t^{2}-|x|^{2}\right) \frac{\partial}{\partial x_{k}}+x_{k}\left(t \frac{\partial}{\partial t}+\left\langle x, \frac{\partial}{\partial x}\right\rangle\right), 1 \leq k \leq 3,
\end{aligned}
$$

and

$$
\frac{\partial}{\partial T}=\frac{1}{2}\left(1+t^{2}-|x|^{2}\right) \frac{\partial}{\partial t}+t\left\langle x, \frac{\partial}{\partial x}\right\rangle
$$

Note that the coefficients of $\partial / \partial T$ and $\partial / \partial Y$ in (2.18) and $(2.20)$ are $O\left((\pi-T)^{2}\right)$ when, say, $0 \leq T<\pi$ and $R \leq(\pi-T) / 4$. Similarly, if $|x| \leq t / 4$ the coefficients of $\partial / \partial t$ and $\partial / \partial x$ in $(2.23)$ and (2.24) are $O\left(t^{2}+|x|^{2}\right)$. Hence we have the following useful result:

Proposition 2.2. In the region where $|x| \leq t / 4$ we can write

$$
\frac{\partial}{\partial t}=\sum a_{0 k}(T, X) \Gamma_{k}, \quad \text { and } \quad \frac{\partial}{\partial x_{j}}=\sum a_{j k}(T, X) \Gamma_{k},
$$

where if $P_{0}$ is as in (2.13)

$$
\left|\Gamma^{\alpha} a_{j k}\right| \leq C \operatorname{dist}\left((T, X), P_{0}\right)^{2-|\alpha|}, \quad|\alpha| \leq 2 .
$$

Also, if $0 \leq T<\pi$ and $R \leq(\pi-T) / 4$

$$
\frac{\partial}{\partial T}=b_{00}(t, x) \frac{\partial}{\partial t}+\sum b_{0 k}(t, x) \frac{\partial}{\partial x_{k}}
$$

and

$$
X_{0} \frac{\partial}{\partial X_{j}}-X_{j} \frac{\partial}{\partial X_{0}}=b_{0 j}(t, x) \frac{\partial}{\partial t}+\sum b_{j k}(t, x) \frac{\partial}{\partial x_{j}},
$$

where if $D=\left(\partial / \partial t, \partial / \partial x_{1}, \ldots, \partial / \partial x_{3}\right)$

$$
\left|D^{\alpha} b_{j k}\right| \leq C(1+|(t, x)|)^{2-|\alpha|}, \quad|\alpha| \leq 2 .
$$

We need one more thing before we can state our main inequality. If $u$ is a function on $\overline{\mathbb{E}}_{+}^{1+3}$, as before, let $\tilde{u}$ denote the pullback of $\Omega u$ to $\mathbb{R}^{1+3}$. If we fix a null form $Q$ as in (1.1) or (1.2) we shall let

$$
\begin{aligned}
\mathcal{Q}(u(T, X), d u(T, X) ; v(T, X), d v & (T, X)) \\
& =\Omega^{-3} Q(d \tilde{u}(t, x), d \tilde{v}(t, x)), \quad \mathcal{P}(t, x)=(T, X) .
\end{aligned}
$$


The reason for this is that if we change the notation a bit from the preceding section and write our main equation (1.3) as

$$
\left\{\begin{array}{l}
\tilde{\square} \tilde{u}=Q(d \tilde{u}, d \tilde{u}), \quad(t, x) \in \mathbb{R}_{+} \times \mathbb{R}^{3} \backslash \mathcal{K} \\
\left.\tilde{u}(t, \cdot)\right|_{\partial \mathcal{K}}=0 \\
\tilde{u}(0, \cdot)=\tilde{f}, \quad \partial_{t} \tilde{u}(0, \cdot)=\tilde{g},
\end{array}\right.
$$

then by (2.9) it transforms to the following equation in $\overline{\mathbb{E}}_{+}^{1+3} \backslash \mathcal{K}_{*}$

$$
\left\{\begin{array}{l}
\left(\square_{g}+1\right) u=\mathcal{Q}(u, d u ; u, d u) \\
u(T, X)=0, \quad(T, X) \in \partial \mathcal{K}_{*} \\
u(0, \cdot)=f, \quad \partial_{T} u(0, \cdot)=g,
\end{array}\right.
$$

if the data satisfies $\tilde{f}=\Omega f$ and $\tilde{g}=\Omega^{2} g$ and if $\mathcal{K}_{*}$ is as in (2.11).

We are now in a position to state our main estimate. It can be thought of as an appropriate version of Theorem 1.6 for $\mathbb{E}_{+}^{1+3} \backslash \mathcal{K}_{*}$, where $\mathcal{K}_{*}$ is as above. Also, we shall see in Section 7 that it immediately yields the desired existence results in Theorem 1.1. It involves solutions of the Dirichlet-wave equation in $\mathbb{E}_{+}^{1+3} \backslash \mathcal{K}_{*}$ :

$$
\left\{\begin{array}{l}
\left(\square_{g}+1\right) u=F \\
\left.u\right|_{\partial \mathcal{K}_{*}}=0 \\
\left.u\right|_{T=0}=u_{0},\left.\quad \partial_{T} u\right|_{T=0}=u_{1} .
\end{array}\right.
$$

Theorem 2.3. Suppose that $u$ solves (2.28) and that $v$ solves the same equation with $u_{0}, u_{1}$ and $F$ replaced by $v_{0}, v_{1}$ and $G$, respectively. If $P_{0}$ is as in (2.13), then

$$
\begin{aligned}
& \sum_{|\alpha| \leq 1}\left\|\left(\operatorname{dist}^{2}\left(P, P_{0}\right) \Gamma\right)^{\alpha} \mathcal{Q}(u, d u ; v, d v)\right\|_{L^{2}\left(\mathbb{E}_{+}^{1+3} \backslash \mathcal{K}_{*}\right)} \\
& \leq C\left(\left\|u_{0}\right\|_{H_{D}^{2}}+\left\|u_{1}\right\|_{H_{D}^{1}}+\sum_{|\alpha| \leq 1}\left\|\left(\operatorname{dist}^{2}\left(P, P_{0}\right) \Gamma\right)^{\alpha} F\right\|_{L^{2}\left(\mathbb{E}_{+}^{1+3} \backslash \mathcal{K}_{*}\right)}\right) \\
& \quad \times\left(\left\|v_{0}\right\|_{H_{D}^{2}}+\left\|v_{1}\right\|_{H_{D}^{1}}+\sum_{|\alpha| \leq 1}\left\|\left(\operatorname{dist}^{2}\left(P, P_{0}\right) \Gamma\right)^{\alpha} G\right\|_{L^{2}\left(\mathbb{E}_{+}^{1+3} \backslash \mathcal{K}_{*}\right)}\right) .
\end{aligned}
$$

Furthermore, the following estimate holds

$$
\begin{aligned}
& \sum_{|\alpha| \leq 1}\left\|\operatorname{dist}^{2|\alpha|}\left(P, P_{0}\right) \Gamma^{\alpha} u(T, \cdot)\right\|_{L^{8}\left(\mathbb{E}_{+}^{1+3} \backslash \mathcal{K}_{*}\right)} \\
& \leq C\left(\left\|u_{0}\right\|_{H_{D}^{2}}+\left\|u_{1}\right\|_{H_{D}^{1}}+\sum_{|\alpha| \leq 1}\left\|\left(\operatorname{dist}^{2}\left(P, P_{0}\right) \Gamma\right)^{\alpha} F\right\|_{L^{2}\left(\mathbb{E}_{+}^{1+3} \backslash \mathcal{K}_{*}\right)}\right) .
\end{aligned}
$$

In many ways (2.29) is a natural extension of (1.17) to the current setting. This is because, near $\partial \mathcal{K}_{*}$, Minkowski derivatives $\partial / \partial x_{j}, 0 \leq j \leq 3$ are pushed forward via $\mathcal{P}$ to ones in $\overline{\mathbb{E}}^{1+3} \backslash \mathcal{K}_{*}$ that behave like linear combinations of the $\Gamma$ with $O\left(\operatorname{dist}^{2}\left(P, P_{0}\right)\right)$ coefficients. In view of (2.14), the weights in (2.29) also naturally compensate for the degeneracy of $\partial \mathcal{K}_{*}$ as $T \rightarrow \pi$.

The proof of (2.29) will require more precise information about the behavior of $\mathcal{Q}$ as $P \rightarrow P_{0}$. The result we shall need says in part that $\mathcal{Q}$ involves the standard null forms 
on $\mathbb{R} \times S^{3}$. These, we recall (see [2], [20], [3]) are

$$
\bar{Q}_{0}(d u, d v)=\sum g^{\mu \nu}(T, X) u_{\mu} v_{\nu}
$$

and

$$
\bar{Q}_{j k}(d u, d v)=\Gamma_{j} u \Gamma_{k} v-\Gamma_{k} u \Gamma_{j} v
$$

where $u_{\mu}$ denotes the differential of $u, g^{\mu \nu}$ is the cometric associated with $g$, and $\Gamma_{j}$, $0 \leq j \leq 6$, are as above.

With this notation, we have the following

Proposition 2.4. Fix a $\mathbb{R}^{1+3}$ null form $Q$ in (1.1)-(1.2) and let $\mathcal{Q}(u, d u ; v, d v)$ be defined as in (2.25). Then $\mathcal{Q}$ extends to a bilinear function of $(u, d v ; v, d v)$ on $\mathbb{R} \times S^{3}$ with smooth coefficients. Moreover, we can write

$$
\mathcal{Q}=a_{0} \bar{Q}_{0}(d u, d v)+\sum_{j k} a_{j k} \bar{Q}_{j k}(d u, d v)+u \sum_{|\alpha|=1} b_{1, \alpha} \Gamma^{\alpha} v+v \sum_{|\alpha|=1} b_{2, \alpha} \Gamma^{\alpha} u+c(T, X) u v,
$$

where the coefficients are smooth, and, moreover,

$$
\Gamma^{\alpha} a_{0}, \Gamma^{\alpha} a_{j k}=O\left(\operatorname{dist}^{2-|\alpha|}\left(P, P_{0}\right)\right), \quad 0 \leq|\alpha| \leq 2,
$$

and

$$
b_{j, \alpha}=O\left(\operatorname{dist}\left(P, P_{0}\right)\right) .
$$

The proof of Proposition 2.4 has two steps. The hard step was carried out by Christodoulou [2] who showed that one can write $\mathcal{Q}$ as in (2.31) with the coefficients $a_{0}, a_{j k}, b_{j, \alpha}$, and $c$ being smooth. Given this, the next step is to observe that Proposition 2.2 implies that if we restrict the coefficients to the region where $R \leq(\pi-T) / 4$, then the $a_{0}$ and $a_{j k}$ must vanish to second order at $P_{0}$ while the $b_{j, \alpha}$ must vanish to first order there. If we combine the two steps, we conclude that they have this order of vanishing at $P_{0}$ when regarded as functions of $\mathbb{R} \times S^{3}$, which completes the proof.

To conclude this section, we show that, in order to establish (2.29), it suffices to consider the case where the Cauchy data vanishes, that is, $u_{0}=u_{1}=0, v_{0}=v_{1}=0$, and where $F$ and $G$ vanish for $T$ near 0 . To see this, fix $0<\delta \leq 1$ and $R_{0}<\pi$ so that $\mathcal{K}_{*} \subset\left\{R<R_{0}-\delta\right\}$. On the set $[0, \delta] \times S^{3}$, the function $\operatorname{dist}\left(P, P_{0}\right)$ is bounded from below, and thus the estimate (2.29) restricted to this set is implied by the following

$$
\begin{aligned}
& \sum_{|\alpha| \leq 1}\left\|\left(\operatorname{dist}^{2}\left(P, P_{0}\right) \Gamma\right)^{\alpha} \mathcal{Q}\right\|_{L^{2}\left([0, \delta] \times S^{3} \backslash \mathcal{K}_{*}\right)} \\
& \leq C\left(\left\|u_{0}\right\|_{H_{D}^{2}}+\left\|u_{1}\right\|_{H_{D}^{1}}+\sum_{|\alpha| \leq 1}\left\|\left(\operatorname{dist}^{2}\left(P, P_{0}\right) \Gamma\right)^{\alpha} F\right\|_{L^{2}\left(\mathbb{E}_{+}^{1+3} \backslash \mathcal{K}_{*}\right)}\right) \\
& \quad \times\left(\left\|v_{0}\right\|_{H_{D}^{2}}+\left\|v_{1}\right\|_{H^{1}}+\sum_{|\alpha| \leq 1}\left\|\left(\operatorname{dist}^{2}\left(P, P_{0}\right) \Gamma\right)^{\alpha} G\right\|_{L^{2}\left(\mathbb{E}_{+}^{1+3} \backslash \mathcal{K}_{*}\right)}\right) .
\end{aligned}
$$

This estimate is established by separately considering the set $R<R_{0}$, on which it follows by the Minkowski estimate (1.17), and the set $R>R_{0}$, on which it holds by the local estimates of [20]. Now fix a function $\psi=\psi(T)$ which vanishes near 0 , and $\psi=1$ for $T \geq \delta$. It then suffices to establish $(2.29)$ with $u$ replaced by $\psi u$, which vanishes near $T=0$, and $F$ replaced by $\psi F+\left[\square_{g}, \psi\right] u$. By combining Minkowski energy estimates 
on $R<R_{0}$ with $\mathbb{E}_{+}^{1+3}$ energy estimates on $R>R_{0}$, together with the fact that $\psi^{\prime}$ is supported in $[0, \delta]$, we obtain the bound

$$
\begin{aligned}
& \sum_{|\alpha| \leq 1}\left\|\left(\operatorname{dist}^{2}\left(P, P_{0}\right) \Gamma\right)^{\alpha}\left(\psi F+\left[\square_{g}, \psi\right] u\right)\right\|_{L^{2}\left(\mathbb{E}_{+}^{1+3} \backslash \mathcal{K}_{*}\right)} \\
& \leq C\left(\left\|u_{0}\right\|_{H_{D}^{2}}+\left\|u_{1}\right\|_{H_{D}^{1}}+\sum_{|\alpha| \leq 1}\left\|\left(\operatorname{dist}^{2}\left(P, P_{0}\right) \Gamma\right)^{\alpha} F\right\|_{L^{2}\left(\mathbb{E}_{+}^{1+3} \backslash \mathcal{K}_{*}\right)}\right) .
\end{aligned}
$$

Similar comments hold for $v$. This completes the reduction of (2.29) to the case of vanishing Cauchy data.

\section{Unit neighborhoods of the obstacle in Minkowski space.}

In this section and the next, we establish the part of (2.29) (with vanishing Cauchy data) where the norm on the left hand side is taken over a set in the Einstein diamond corresponding to a unit neighborhood of the obstacle in Minkowski space. Precisely, we fix $A>0$ so that $\mathcal{K} \subset|x| \leq A / 2$. Let

$$
Y_{+}=\mathcal{P}\left(\left\{(t, x): t>0, x \in \mathbb{R}^{3} \backslash \mathcal{K} \text { and }|x|<A\right\}\right) .
$$

We similarly define $Y_{+}^{*}$ and $Y_{+}^{* *}$ by replacing $A$ by $2 A$ and $3 A$ respectively. In a neighborhood of the tip $P_{0}=(\pi, 0)$ of the Einstein diamond, $Y_{+}$has the form

$$
Y_{+}=\left\{(T, X) \in \mathbb{E}_{+}^{1+3} \backslash \mathcal{K}_{*}: R \leq A_{0}(T)(\pi-T)^{2}\right\}
$$

where $A_{0}(T)$ is smooth and nonvanishing at $P_{0}$. Also, on $Y_{+}$, we have

$$
\Omega \approx(\pi-T)^{2} \approx(1+t)^{-2}
$$

Similar comments hold for $Y_{+}^{*}$ and $Y_{+}^{* *}$. We shall establish estimates on $Y_{+}$by pulling them back to Minkowski space. The following will play a key role.

Proposition 3.1. Let $\tilde{\square} \tilde{u}=\tilde{F}$ and $\tilde{\square} \tilde{v}=\tilde{G}$ be solutions of the Dirichlet-wave equation in $\mathbb{R}_{+} \times \mathbb{R}^{3} \backslash \mathcal{K}$ with vanishing Cauchy data. Assume that $\tilde{F}(t, x)=\tilde{G}(t, x)=0$ when $|x|>2 A$. Then if $N \geq 0$ is fixed,

$$
\begin{aligned}
& \sum_{|\alpha| \leq 1}\left\|(1+t)^{N} D^{\alpha} Q(d \tilde{u}, d \tilde{v})\right\|_{L^{2}(|x|<2 A, t>0)} \\
& \leq C \sum_{|\alpha| \leq 1}\left\|(1+t)^{N / 2} D^{\alpha} \tilde{F}\right\|_{L^{2}(d x d t)} \sum_{|\alpha| \leq 1}\left\|(1+t)^{N / 2} D^{\alpha} \tilde{G}\right\|_{L^{2}(d x d t)}
\end{aligned}
$$

We are using the notation of the preceding section. In particular $\tilde{\square}=\partial^{2} / \partial t^{2}-\Delta$ is the standard D'Alembertian in Minkowski space, and $D=\left(\partial / \partial t, \ldots, \partial / \partial x_{3}\right)$.

Proposition 3.1 follows in a straightforward way from Proposition 1.5 and Theorem 1.6. Before giving the simple proof, let us see its relevance for (2.29).

We shall apply (3.1) when $N=2$. We observe that we have the following estimate, if $\tilde{u}=\mathcal{P}^{*}(\Omega u)$ and $\tilde{v}=\mathcal{P}^{*}(\Omega v)$

$$
\sum_{|\alpha| \leq 1}\left\|\left(\operatorname{dist}^{2}\left(P, P_{0}\right) \Gamma\right)^{\alpha} \mathcal{Q}\right\|_{L^{2}\left(Y_{+}\right)} \leq C \sum_{|\alpha| \leq 1}\left\|(1+t)^{2} D^{\alpha} Q(d \tilde{u}, d \tilde{v})\right\|_{L^{2}(|x|<A, t>0)} .
$$


This is easy to verify. One first notes that the usual measure on $\mathbb{E}^{1+3}$ gets pulled back to $\Omega^{4} d x d t$ in view of (2.6). Also, $\operatorname{dist}^{2}\left(P, P_{0}\right) \approx \Omega$ in $Y_{+}$. Therefore, Proposition 2.2, (2.7), and (2.25) imply that the left side of (3.2) is controlled by

$$
\sum_{|\alpha| \leq 1}\left\|\Omega^{2} D^{\alpha}\left[\Omega^{-3} Q(d \tilde{u}, d \tilde{v})\right]\right\|_{\left.L^{2}(|x|<A, t>0)\right)} \leq C \sum_{|\alpha| \leq 1}\left\|\Omega^{-1} D^{\alpha} Q(d \tilde{u}, d \tilde{v})\right\|_{\left.L^{2}(|x|<A, t>0)\right)} .
$$

Since $\Omega^{-1}=O\left((1+t)^{2}\right)$ in $Y_{+}$, this yields our assertion (3.2).

To proceed, we first recall that

$$
\tilde{\square} \tilde{u}=\tilde{F}=\Omega^{3} F .
$$

We cannot apply (3.1), though, since $\tilde{F}$ does not have the required support property. To get around this, choose $\eta \in C_{0}^{\infty}\left(\mathbb{R}^{3}\right)$ satisfying $\eta(x)=1$ if $|x|<A$ and $\eta(x)=0$ if $|x|>2 A$. We then let $\tilde{u}^{1}=\eta \tilde{u}$, and note that

$$
\tilde{\square} \tilde{u}^{1}=2 \nabla_{x} \eta \cdot \nabla_{x} \tilde{u}+\left(\Delta_{x} \eta\right) \tilde{u}+\eta \tilde{F}=\tilde{F}^{1}
$$

has the required support properties. Also, note that $\tilde{u}^{1}=\tilde{u}$ when $|x| \leq A$.

If we do the same for $\tilde{v}$, then (3.1) and (3.2) imply that

$$
\begin{aligned}
\sum_{|\alpha| \leq 1} \| & \left(\operatorname{dist}^{2}\left(P, P_{0}\right) \Gamma\right)^{\alpha} \mathcal{Q}(u, d u ; v, d v) \|_{L^{2}\left(Y_{+}\right)} \\
& \leq C\left(\sum_{|\alpha| \leq 1}\left\|(1+t) D^{\alpha} \tilde{F}^{1}\right\|_{L^{2}(d x d t)}\right)\left(\sum_{|\alpha| \leq 1}\left\|(1+t) D^{\alpha} \tilde{G}^{1}\right\|_{L^{2}(d x d t)}\right) \\
\leq & C\left(\sum_{|\alpha| \leq 1}\left\|(1+t) D^{\alpha} F\right\|_{L^{2}(|x| \leq 2 A, t>0)}+\sum_{|\alpha| \leq 2}\left\|(1+t) D^{\alpha} u\right\|_{L^{2}(|x| \leq 2 A, t>0)}\right) \\
& \times\left(\sum_{|\alpha| \leq 1}\left\|(1+t) D^{\alpha} G\right\|_{L^{2}(|x| \leq 2 A, t>0)}+\sum_{|\alpha| \leq 2}\left\|(1+t) D^{\alpha} v\right\|_{L^{2}(|x| \leq 2 A, t>0)}\right) .
\end{aligned}
$$

If we repeat the proof of (3.2), we conclude that the term involving $F$ in the right is controlled by

$$
\sum_{|\alpha| \leq 1}\left\|(\pi-T)^{-1} \Omega^{|\alpha|} \Omega^{3} \Omega^{-2} \Gamma^{\alpha} F\right\|_{L^{2}\left(Y_{+}^{*}\right)} \approx \sum_{|\alpha| \leq 1}\left\|\operatorname{dist}\left(P, P_{0}\right)\left(\operatorname{dist}^{2}\left(P, P_{0}\right) \Gamma\right)^{\alpha} F\right\|_{L^{2}\left(Y_{+}^{*}\right)} .
$$

Similarly, the term involving $\tilde{u}$ in the right side of (3.3) is dominated by

$$
\sum_{|\alpha| \leq 1}\left\|\operatorname{dist}^{1+2(|\alpha|-2)}\left(P, P_{0}\right) \Gamma^{\alpha} u\right\|_{L^{2}\left(Y_{+}^{*}\right)} .
$$

Putting all of this together gives us the following

\section{Proposition 3.2.}

$$
\begin{aligned}
& \sum_{|\alpha| \leq 1}\left\|\left(\operatorname{dist}^{2}\left(P, P_{0}\right) \Gamma\right)^{\alpha} \mathcal{Q}\right\|_{L^{2}\left(Y_{+}\right)} \\
& \quad \leq C\left(\sum_{|\alpha| \leq 1}\left\|d i s t^{1+2|\alpha|}\left(P, P_{0}\right) \Gamma^{\alpha} F\right\|_{L^{2}\left(Y_{+}^{*}\right)}+\sum_{|\alpha| \leq 2}\left\|d i s t^{1+2(|\alpha|-2)}\left(P, P_{0}\right) \Gamma^{\alpha} u\right\|_{L^{2}\left(Y_{+}^{*}\right)}\right) \\
& \times\left(\sum_{|\alpha| \leq 1}\left\|d i s t^{1+2|\alpha|}\left(P, P_{0}\right) \Gamma^{\alpha} G\right\|_{L^{2}\left(Y_{+}^{*}\right)}+\sum_{|\alpha| \leq 2}\left\|d i s t^{1+2(|\alpha|-2)}\left(P, P_{0}\right) \Gamma^{\alpha} v\right\|_{L^{2}\left(Y_{+}^{*}\right)}\right) .
\end{aligned}
$$


Proof of Proposition 3.1. We first note that for $j=1,2,3, \ldots$ the local estimate (1.17) gives

$$
\begin{aligned}
\sum_{|\alpha| \leq 1}\left\|(1+t)^{N} D^{\alpha} Q(d \tilde{u}, d \tilde{v})\right\|_{L^{2}(|x|<2 A, t \in[j-1, j])} & \\
\leq & C\left((1+j)^{N / 2}\left[\|\tilde{u}(j, \cdot)\|_{H_{D}^{2}(|x|<2 A+1)}+\left\|\partial_{t} \tilde{u}(j, \cdot)\right\|_{H_{D}^{1}(|x|<2 A+1)}\right]\right. \\
& \left.+\sum_{|\alpha| \leq 1}\left\|(1+t)^{N / 2} D^{\alpha} \tilde{F}\right\|_{L^{2}(\{(t, x): t \in[j-1, j]\})}\right) \\
& \times\left((1+j)^{N / 2}\left[\|\tilde{v}(j, \cdot)\|_{H_{D}^{2}(|x|<2 A+1)}+\left\|\partial_{t} \tilde{v}(j, \cdot)\right\|_{H_{D}^{1}(|x|<2 A+1)}\right]\right. \\
& \left.+\sum_{|\alpha| \leq 1}\left\|(1+t)^{N / 2} D^{\alpha} \tilde{G}\right\|_{L^{2}(\{(t, x): t \in[j-1, j]\})}\right) .
\end{aligned}
$$

On the other hand, the decay estimates (1.16), yields

$$
\begin{aligned}
(1+j)^{N / 2}\left(\|\tilde{u}(j, \cdot)\|_{H_{D}^{2}(|x|<2 A+1)}\right. & \left.+\left\|\partial_{t} \tilde{u}(j, \cdot)\right\|_{H_{D}^{1}(|x|<2 A+1)}\right) \\
& \leq C_{N} \sum_{|\alpha| \leq 1}\left\|e^{-c_{0}(j-t)}(1+t)^{N / 2} D^{\alpha} \tilde{F}\right\|_{L^{2}(\{(t, x): t<j\}),}
\end{aligned}
$$

for some $c_{0}>0$. The same estimate works for the other factor in the right side of (3.5).

If we combine the last two sets of inequalities and square the left side we get

$$
\begin{aligned}
& \sum_{|\alpha| \leq 1}\left\|(1+t)^{N} D^{\alpha} Q(d \tilde{u}, d \tilde{v})\right\|_{L^{2}(|x|<2 A, t \in[j-1, j])}^{2} \\
& \leq C_{N} \sum_{1 \leq i \leq j} \sum_{|\alpha| \leq 1} e^{-2 c_{0}(j-i)}\left\|(1+t)^{N / 2} D^{\alpha} \tilde{F}\right\|_{L^{2}\left([i-1, i] \times \mathbb{R}^{3} \backslash \mathcal{K}\right)}^{2} \\
& \times \sum_{1 \leq i \leq j} \sum_{|\alpha| \leq 1} e^{-2 c_{0}(j-i)}\left\|(1+t)^{N / 2} D^{\alpha} \tilde{G}\right\|_{L^{2}\left([i-1, i] \times \mathbb{R}^{3} \backslash \mathcal{K}\right)}^{2}
\end{aligned}
$$

If we sum this inequality over $j$, we obtain (3.1).

\section{Localized energy estimates.}

We shall be able to handle the terms in (3.4) involving $\Gamma^{\alpha} u$ and $\Gamma^{\alpha} v$ using the following

Proposition 4.1. If $u$ and $F$ are as in (2.28), and $u_{0}=u_{1}=0$, then

$$
\sum_{|\alpha| \leq 2}\left\|\operatorname{dist} t^{1+2(|\alpha|-2)}\left(P, P_{0}\right) \Gamma^{\alpha} u\right\|_{L^{2}\left(Y_{+}^{*}\right)} \leq C \sum_{|\alpha| \leq 1}\left\|\left(\operatorname{dist}^{2}\left(P, P_{0}\right) \Gamma\right)^{\alpha} F\right\|_{L^{2}\left(\overline{\mathbb{E}}_{+}^{1+3}\right)} .
$$

If we combine this with Proposition 3.2 we conclude that the analog of (2.29) holds if on the left hand side the norm is taken over $Y_{+}$. of

To prove Proposition 4.1 we require a couple of Minkowski space estimates for solutions

$$
\left\{\begin{array}{l}
\tilde{\square} \tilde{u}(t, x)=\tilde{F}(t, x), \quad(t, x) \in \mathbb{R}_{+} \times \mathbb{R}^{3} \backslash \mathcal{K} \\
\tilde{u}(t, x)=0, \quad x \in \partial \mathcal{K} \\
\tilde{u}(0, x)=0, \quad \partial_{t} \tilde{u}(0, x)=0 .
\end{array}\right.
$$


The first estimate we need follows immediately from (1.16). It says that if $N>0$ and $A<+\infty$ are fixed then

$$
\begin{aligned}
& \sum_{|\alpha| \leq 2}\left\|(1+t)^{N} D^{\alpha} \tilde{u}\right\|_{L^{2}(|x|<2 A, t>0)} \leq C \sum_{|\alpha| \leq 1}\left\|(1+t)^{N} D^{\alpha} \tilde{F}\right\|_{L^{2}(d x d t)} \\
& \quad \text { if } \tilde{F}(t, x)=0, \text { when }|x|>2 A .
\end{aligned}
$$

We shall also require the following Minkowski space estimate which is useful when $\tilde{F}$ vanishes near the boundary.

Proposition 4.2. Suppose that $\tilde{u}$ solves (4.2). Assume further that $\tilde{F}(t, x)=0$ when $|x|<5 A / 2$. Let $\tilde{\square} \tilde{u}_{f}=\tilde{F}$ be the solution of the corresponding free wave equation. Then given fixed $N>0$

$$
\sum_{|\alpha| \leq 2}\left\|(1+t)^{N} D^{\alpha} \tilde{u}\right\|_{L^{2}(|x|<2 A, t>0)} \leq C \sum_{|\alpha| \leq 2}\left\|(1+t)^{N} D^{\alpha} \tilde{u}_{f}\right\|_{L^{2}(|x|<5 A / 2, t>0)} .
$$

To handle $u_{f}$ in (4.4) we shall use the following result. As we shall see it follows easily from Huygen's principle and standard estimates for the free wave equation.

Lemma 4.3. Let $\tilde{u}_{f}$ solve the free (no obstacle) wave equation $\square u_{f}=F$ on the Einstein cylinder, with zero Cauchy data. Then

$$
\sum_{|\alpha| \leq 2}\left\|d i s t^{1+2(|\alpha|-2)}\left(P, P_{0}\right) \Gamma^{\alpha} u_{f}\right\|_{L^{2}\left(Y_{+}^{* *}\right)} \leq C \sum_{|\alpha| \leq 1}\left\|\left(\operatorname{dist}^{2}\left(P, P_{0}\right) \Gamma\right)^{\alpha} F\right\|_{L^{2}\left(\overline{\mathbb{E}}_{+}^{1+3}\right)} .
$$

Let us momentarily postpone the proofs and see how (4.3)-(4.5) can be used to prove Proposition 4.1.

For this we first notice that the arguments giving (3.2) imply that

$$
\sum_{|\alpha| \leq 2}\left\|\operatorname{dist}^{1+2(|\alpha|-2)}\left(P, P_{0}\right) \Gamma^{\alpha} u\right\|_{L^{2}\left(Y_{+}^{*}\right)} \leq C \sum_{|\alpha| \leq 2}\left\|(1+t) D^{\alpha} \tilde{u}\right\|_{L^{2}(|x|<2 A, t>0)} .
$$

Here $u$ and $\tilde{u}$ are identified as above.

We then fix $\theta \in C_{0}^{\infty}\left(\mathbb{R}^{3}\right)$ satisfying $\theta(x)=1$ if $|x| \leq 5 A / 2$ and $\theta(x)=0$ if $|x| \geq 3 A$. Using $\theta$ we split

$$
\tilde{F}=\theta \tilde{F}+(1-\theta) \tilde{F}=\tilde{G}+\tilde{H}
$$

We then decompose $\tilde{u}=\tilde{v}+\tilde{w}$, where $\tilde{\square} \tilde{v}=\tilde{G}$. Note then that the forcing term $\tilde{H}$ for $\tilde{w}$ vanishes for $|x| \leq 5 A / 2$. Also, the preceding inequality yields

$$
\begin{aligned}
& \sum_{|\alpha| \leq 2}\left\|\operatorname{dist}^{1+2(|\alpha|-2)}\left(P, P_{0}\right) \Gamma^{\alpha} u\right\|_{L^{2}\left(Y_{+}^{*}\right)} \\
& \quad \leq C \sum_{|\alpha| \leq 2}\left\|(1+t) D^{\alpha} \tilde{v}\right\|_{L^{2}(|x|<2 A, t>0)}+C \sum_{|\alpha| \leq 2}\left\|(1+t) D^{\alpha} \tilde{w}\right\|_{L^{2}(|x|<2 A, t>0)}
\end{aligned}
$$


If we use (4.3) we get that

$$
\begin{aligned}
\sum_{|\alpha| \leq 2}\left\|(1+t) D^{\alpha} \tilde{v}\right\|_{L^{2}(|x|<2 A, t>0)} & \leq C \sum_{|\alpha| \leq 1}\left\|(1+t) D^{\alpha} \tilde{G}\right\|_{L^{2}(d x d t)} \\
& \leq C \sum_{|\alpha| \leq 1}\left\|(1+t) D^{\alpha} \tilde{F}\right\|_{L^{2}(|x|<3 A, t>0)} \\
& \leq C \sum_{|\alpha| \leq 1}\left\|\operatorname{dist}^{1+2|\alpha|}\left(P, P_{0}\right) \Gamma^{\alpha} F\right\|_{L^{2}\left(Y_{+}^{* *}\right)}
\end{aligned}
$$

Similarly, if we use Proposition 4.2 we get that

$$
\sum_{|\alpha| \leq 2}\left\|(1+t) D^{\alpha} \tilde{w}\right\|_{L^{2}(|x|<2 A, t>0)} \leq C \sum_{|\alpha| \leq 2}\left\|(1+t) D^{\alpha} \tilde{w}_{f}\right\|_{L^{2}(|x|<3 A, t>0)},
$$

if $\tilde{\square} \tilde{w}_{f}=\tilde{H}$ is the solution of the free wave equation. If then $w_{f}$ and $H$ denote the images of $\Omega^{-1} \tilde{w}_{f}$ and $\Omega^{-3} \tilde{H}$ in the Einstein diamond, then Lemma 4.3 and the above arguments yield

$$
\begin{aligned}
\sum_{|\alpha| \leq 2}\left\|(1+t) D^{\alpha} \tilde{w}_{f}\right\|_{L^{2}(|x|<3 A, t>0)} & \leq C \sum_{|\alpha| \leq 2}\left\|\operatorname{dist}^{1+2(|\alpha|-2)}\left(P, P_{0}\right) \Gamma^{\alpha} w_{f}\right\|_{L^{2}\left(Y_{+}^{* *}\right)} \\
& \leq C \sum_{|\alpha| \leq 1}\left\|\left(\operatorname{dist}^{2}\left(P, P_{0}\right) \Gamma\right)^{\alpha} H\right\|_{L^{2}\left(\overline{\mathbb{E}}_{+}^{1+3}\right)} \\
& \leq C \sum_{|\alpha| \leq 1}\left\|\left(\operatorname{dist}^{2}\left(P, P_{0}\right) \Gamma\right)^{\alpha} F\right\|_{L^{2}\left(\overline{\mathbb{E}}_{+}^{1+3}\right)}
\end{aligned}
$$

In the last step we used the fact that

$$
\sum_{|\alpha|=1}\left|\Gamma^{\alpha} H\right| \leq C \sum_{|\alpha| \leq 1} \operatorname{dist}^{2|\alpha|-2}\left(P, P_{0}\right)\left|\Gamma^{\alpha} F\right| .
$$

Combining (4.6)-(4.9) yields Proposition 4.1.

Proof of Proposition 4.2. Let us first write

$$
\tilde{u}=\tilde{u}_{f}-\tilde{u}_{r}
$$

where $\tilde{u}_{f}$ is the solution to the free (i.e., boundaryless) wave equation $\tilde{\square} \tilde{u}_{f}=\tilde{F}$ in $\mathbb{R}_{+}^{1+3}$, and where $\tilde{u}_{r}$ is the reflection term.

To make use of the support assumptions, let us fix $\rho \in C_{0}^{\infty}\left(\mathbb{R}^{3}\right)$ satisfying $\rho(x)=1$ if $|x|<2 A$ and $\rho(x)=0$ if $|x|>5 A / 2$. Then clearly

$$
\tilde{u}(t, x)=\rho(x) \tilde{u}_{f}(t, x)-\tilde{u}_{r}(t, x) \quad \text { if }|x|<2 A .
$$

We next observe that $\rho \tilde{u}_{f}-\tilde{u}_{r}$ vanishes on $\mathbb{R}_{+} \times \partial \mathcal{K}$, and

$$
\tilde{\square}\left(\rho \tilde{u}_{f}-\tilde{u}_{r}\right)=\rho \tilde{F}-2 \nabla_{x} \rho \cdot \nabla_{x} \tilde{u}_{f}-\left(\Delta_{x} \rho\right) \tilde{u}_{f}=-2 \nabla_{x} \rho \cdot \nabla_{x} \tilde{u}_{f}-\left(\Delta_{x} \rho\right) \tilde{u}_{f},
$$

since the support assumptions imply that $\rho \tilde{F}=0$. For the sake of notation, let $\tilde{G}$ denote the right side of this equation and also set $\tilde{w}=\rho \tilde{u}_{f}-\tilde{u}_{r}$. Note that $\tilde{G}(t, x)=0$ if $|x|>5 A / 2$. 
We now argue as in the proof of Proposition 3.1. If $j=0,1, \ldots$ then $(4.10)$ and (1.16) yield

$$
\begin{aligned}
\sum_{|\alpha| \leq 2}\left\|D^{\alpha} \tilde{u}\right\|_{L^{2}(|x|<2 A, t \in[j, j+1])} & =\sum_{|\alpha| \leq 2}\left\|D^{\alpha} \tilde{w}\right\|_{L^{2}(|x|<2 A, t \in[j, j+1])} \\
& \leq C \sum_{|\alpha| \leq 1}\left\|e^{-c(j+1-t)} D^{\alpha} \tilde{G}\right\|_{L^{2}(\{(t, x): 0<t<j+1\})}
\end{aligned}
$$

This yields (1.4) since

$$
\sum_{|\alpha| \leq 1}\left\|D^{\alpha} \tilde{G}(t, \cdot)\right\|_{L^{2}(d x)} \leq C \sum_{|\alpha| \leq 2}\left\|D^{\alpha} \tilde{u}_{f}(t, \cdot)\right\|_{L^{2}(|x|<5 A / 2)} .
$$

Proof of Lemma 4.3. Write $[0, \pi)=\cup_{j>0} I_{j}$ where $I_{j}$ are intervals $\left[a_{j}, b_{j}\right]$ with $a_{j+1}=b_{j}$ and

$$
\left|I_{j}\right| \approx\left(\pi-b_{j}\right)^{2}
$$

Given $I_{j}$ let

$$
\Lambda_{j}=\left\{(T, X) \in \overline{\mathbb{E}}_{+}^{1+3}: T+R \in I_{j}\right\},
$$

where, as in Section 2, $R$ is the distance from $X$ to the north pole. Then if $T \in I_{j}$, by Huygen's principle, the energy inequality, and the fact that the $\Gamma_{j}$ commute with the D'Alembertian, there is a uniform constant $B$, depending on $A$, so that

$$
\sum_{|\alpha|=2}\left\|\Gamma^{\alpha} u_{f}\right\|_{L^{\infty} L^{2}\left(Y_{+}^{* *}: T \in I_{j}\right)} \leq C \sum_{|k-j|<B} \sum_{|\alpha|=1}\left\|\Gamma^{\alpha} F\right\|_{L^{1} L^{2}\left(\Lambda_{k}\right)} .
$$

By the Schwarz inequality we can dominate the first term on the right by

$$
\begin{aligned}
& \sum_{|k-j|<B} \sum_{|\alpha|=1}\left\|\operatorname{dist}^{2}\left(P, P_{0}\right) \Gamma^{\alpha} F\right\|_{L^{2}\left(\Lambda_{k}\right)} \times\left(\int_{0}^{T}(\pi-s)^{-4} d s\right)^{1 / 2} \\
& \quad \leq C \sum_{|k-j|<B} \sum_{|\alpha|=1}(\pi-T)^{-3 / 2}\left\|\operatorname{dist}^{2}\left(P, P_{0}\right) \Gamma^{\alpha} F\right\|_{L^{2}\left(\Lambda_{k}\right)} .
\end{aligned}
$$

Using these inequalities, we conclude that

$$
\begin{aligned}
\sum_{|\alpha|=2}\left\|\operatorname{dist}\left(P, P_{0}\right) \Gamma^{\alpha} u_{f}\right\|_{L^{2}\left(Y_{+}^{* *}: T \in I_{j}\right)} \leq & C(\pi-T)^{2} \sum_{|\alpha|=2}\left\|\Gamma^{\alpha} u_{f}\right\|_{L^{\infty} L^{2}\left(Y_{+}^{* *}: T \in I_{j}\right)} \\
& \leq C \sum_{|k-j|<B} \sum_{|\alpha|=1}\left\|\operatorname{dist}^{5 / 2}\left(P, P_{0}\right) \Gamma^{\alpha} F\right\|_{L^{2}\left(\Lambda_{k}\right)} .
\end{aligned}
$$

From this we deduce that

$$
\sum_{|\alpha|=2}\left\|\operatorname{dist}\left(P, P_{0}\right) \Gamma^{\alpha} u_{f}\right\|_{L^{2}\left(Y_{+}^{* *}\right)} \leq C \sum_{|\alpha|=1}\left\|\operatorname{dist}^{5 / 2}\left(P, P_{0}\right) \Gamma^{\alpha} F\right\|_{L^{2}\left(\overline{\mathbb{E}}_{+}^{1+3}\right)}
$$

which shows that the terms on the left hand side of (4.5) corresponding to $|\alpha|=2$ satisfy stronger estimates than those asserted by the Lemma.

The same bounds hold for the terms involving $\Gamma^{\alpha} u_{f},|\alpha|=1$. To see this, we first use Hölder's inequality to see that if $T$ is fixed, then

$$
\left\|\operatorname{dist}^{-1}\left(P, P_{0}\right) \Gamma^{\alpha} u_{f}(T, \cdot)\right\|_{L^{2}\left(X:(X, T) \in Y_{+}^{* *}\right)} \leq C\left\|\operatorname{dist}\left(P, P_{0}\right) \Gamma^{\alpha} u_{f}(T, \cdot)\right\|_{L^{6}\left(X:(X, T) \in Y_{+}^{* *}\right)} .
$$


Next, by the energy inequality and Sobolev embedding, if $\left(\square_{g}+1\right) w=H$ with vanishing Cauchy data then

$$
\|w(T, \cdot)\|_{L^{6}\left(S^{3}\right)} \leq C \int_{0}^{T}\|H(S, \cdot)\|_{L^{2}\left(S^{3}\right)} d S .
$$

Consequently, if we use Huygen's principle and repeat our earlier arguments we find that

$$
\sum_{|\alpha|=1}\left\|\Gamma^{\alpha} u_{f}\right\|_{L^{\infty} L^{6}\left(Y_{+}^{* *}: T \in I_{j}\right)} \leq C \sum_{|k-j|<B} \sum_{|\alpha|=1}\left\|\Gamma^{\alpha} F\right\|_{L^{1} L^{2}\left(\Lambda_{k}\right)} .
$$

Therefore, we can dominate the terms in (4.5) with $|\alpha|=1$ by the same bounds as for $|\alpha|=2$.

It remains to handle the terms with $|\alpha|=0$. We may bound

$$
\left\|\operatorname{dist}\left(P, P_{0}\right)^{-3} u_{f}\right\|_{L^{2}\left(Y_{+}^{* *}: T \in I_{j}\right)}^{2} \leq C\left\|u_{f}\right\|_{L_{T}^{\infty} L_{X}^{6}\left(Y_{+}^{* *}: T \in I_{j}\right)}^{2} \leq C \sum_{|k-j|<B}\|F\|_{L^{2}\left(\Lambda_{k}\right)}^{2} .
$$

Summing over $j$ yields the desired bound, completing the proof of Lemma 4.3.

\section{Complement of image of unit neighborhood of obstacle.}

To handle the complement of $Y_{+}$in the Einstein cylinder, we shall use the following estimates for the free wave equation.

Proposition 5.1. Let $\left(\square_{g}+1\right) u_{f}=F,\left(\square_{g}+1\right) v_{f}=G$ be solutions of the free (no obstacle) wave equation in the Einstein cylinder $\mathbb{E}_{+}^{1+3}$. As before, let

$$
\mathcal{Q}\left(u_{f}, d u_{f} ; v_{f}, d v_{f}\right)=\Omega^{-3} Q\left(d \Omega u_{f}, d \Omega v_{f}\right) .
$$

Then

$$
\begin{aligned}
& \sum_{|\alpha| \leq 1}\left\|\left(\operatorname{dist}^{2}\left(P, P_{0}\right) \Gamma\right)^{\alpha} \mathcal{Q}\right\|_{L^{2}\left(\mathbb{E}_{+}^{1+3}\right)} \\
& \leq C\left(\left\|u_{f}(0, \cdot)\right\|_{H^{2}\left(S^{3}\right)}+\left\|\partial_{T} u_{f}(0, \cdot)\right\|_{H^{1}\left(S^{3}\right)}+\sum_{|\alpha| \leq 1}\left\|\left(\operatorname{dist}^{2}\left(P, P_{0}\right) \Gamma\right)^{\alpha} F\right\|_{L^{1} L^{2}\left(\mathbb{E}_{+}^{1+3}\right)}\right) \\
& \quad \times\left(\left\|v_{f}(0, \cdot)\right\|_{H^{2}\left(S^{3}\right)}+\left\|\partial_{T} v_{f}(0, \cdot)\right\|_{H^{1}\left(S^{3}\right)}+\sum_{|\alpha| \leq 1}\left\|\left(\operatorname{dist}^{2}\left(P, P_{0}\right) \Gamma\right)^{\alpha} G\right\|_{L^{1} L^{2}\left(\mathbb{E}_{+}^{1+3}\right)}\right) .
\end{aligned}
$$

The first step in establishing Proposition 5.1 is to observe that it suffices to consider the case where the Cauchy data of $u_{f}$ and $v_{f}$ vanish; that is, $u_{f}(0, \cdot)=\partial_{T} u_{f}(0, \cdot)=0$, and similarly for $v_{f}$. This follows by a similar (but simpler) reduction to that at the end of Section 2. Consequently, we are reduced to establishing the following estimate, in the case of vanishing Cauchy data,

$$
\begin{aligned}
& \sum_{|\alpha| \leq 1}\left\|\left(\operatorname{dist}^{2}\left(P, P_{0}\right) \Gamma\right)^{\alpha} \mathcal{Q}\right\|_{L^{2}\left(\mathbb{E}_{+}^{1+3}\right)} \\
& \quad \leq C \sum_{|\alpha| \leq 1}\left\|\left(\operatorname{dist}^{2}\left(P, P_{0}\right) \Gamma\right)^{\alpha} F\right\|_{L^{1} L^{2}\left(\mathbb{E}_{+}^{1+3}\right)} \sum_{|\alpha| \leq 1}\left\|\left(\operatorname{dist}^{2}\left(P, P_{0}\right) \Gamma\right)^{\alpha} G\right\|_{L^{1} L^{2}\left(\mathbb{E}_{+}^{1+3}\right)} .
\end{aligned}
$$

We postpone the proof of the estimate (5.1) and state a few consequences. For simplicity, we state the next three estimates in the case of vanishing Cauchy data. The first just 
follows from the Schwarz inequality,

$$
\begin{aligned}
& \sum_{|\alpha| \leq 1}\left\|\left(\operatorname{dist}^{2}\left(P, P_{0}\right) \Gamma\right)^{\alpha} \mathcal{Q}\right\|_{L^{2}\left(\mathbb{E}_{+}^{1+3}\right)} \\
& \quad \leq C \sum_{|\alpha| \leq 1}\left\|\left(\operatorname{dist}^{2}\left(P, P_{0}\right) \Gamma\right)^{\alpha} F\right\|_{L^{2}\left(\mathbb{E}_{+}^{1+3}\right)} \sum_{|\alpha| \leq 1}\left\|\left(\operatorname{dist}^{2}\left(P, P_{0}\right) \Gamma\right)^{\alpha} G\right\|_{L^{2}\left(\mathbb{E}_{+}^{1+3}\right)} .
\end{aligned}
$$

As in the preceding section, we can obtain better weighted estimates when the forcing terms are supported near the boundary. One such estimate that we shall need is the following

$$
\begin{aligned}
& \sum_{|\alpha| \leq 1}\left\|\left(\operatorname{dist}^{2}\left(P, P_{0}\right) \Gamma\right)^{\alpha} \mathcal{Q}\right\|_{L^{2}\left(\mathbb{E}_{+}^{1+3}\right)} \\
& \quad \leq C \sum_{|\alpha| \leq 1}\left\|\operatorname{dist}^{1+2|\alpha|}\left(P, P_{0}\right) \Gamma^{\alpha} F\right\|_{L^{2}\left(\mathbb{E}_{+}^{1+3}\right)} \sum_{|\alpha| \leq 1}\left\|\operatorname{dist}^{2|\alpha|}\left(P, P_{0}\right) \Gamma^{\alpha} G\right\|_{L^{2}\left(\mathbb{E}_{+}^{1+3}\right)}, \\
& \quad \text { if } \operatorname{support}(F) \subseteq Y_{+} .
\end{aligned}
$$

Similarly,

$$
\begin{aligned}
& \sum_{|\alpha| \leq 1}\left\|\left(\operatorname{dist}^{2}\left(P, P_{0}\right) \Gamma\right)^{\alpha} \mathcal{Q}\right\|_{L^{2}\left(\mathbb{E}_{+}^{1+3}\right)} \\
& \quad \leq C \sum_{|\alpha| \leq 1}\left\|\operatorname{dist}^{1+2|\alpha|}\left(P, P_{0}\right) \Gamma^{\alpha} F\right\|_{L^{2}\left(\mathbb{E}_{+}^{1+3}\right)} \sum_{|\alpha| \leq 1}\left\|\operatorname{dist}^{1+2|\alpha|}\left(P, P_{0}\right) \Gamma^{\alpha} G\right\|_{L^{2}\left(\mathbb{E}_{+}^{1+3}\right)}, \quad \text { if } \operatorname{support}(F, G) \subseteq Y_{+} .
\end{aligned}
$$

The proof of (5.3) and (5.4) uses a decomposition that is similar to the one employed in the proof of Lemma 4.3. Let $I_{j}$ be as in the proof of Lemma 4.3, and let $\Psi_{j}$ be a partition of unity such that $\Psi_{j}=1$ on $I_{j}$ and $\Psi_{j}$ is supported in the doubled interval $I_{j}^{*}$. Also, let $\Lambda_{j}^{+}$be the subset of $\mathbb{E}_{+}^{1+3}$ where $T-R \in I_{j}$. Then if $F$ is supported in $Y_{+}$, we can use (5.1) and Huygen's principle to see that

$$
\begin{aligned}
& \sum_{|\alpha| \leq 1}\left\|\left(\operatorname{dist}^{2}\left(P, P_{0}\right) \Gamma\right)^{\alpha} \mathcal{Q}\right\|_{L^{2}\left(\Lambda_{j}^{+}\right)} \\
\leq & C \sum_{|k-j|<B} \sum_{|\alpha| \leq 1}\left\|\left(\operatorname{dist}^{2}\left(P, P_{0}\right) \Gamma\right)^{\alpha}\left(\Psi_{k} F\right)\right\|_{L^{1} L^{2}\left(\mathbb{E}_{+}^{1+3}\right)} \sum_{|\alpha| \leq 1}\left\|\left(\operatorname{dist}^{2}\left(P, P_{0}\right) \Gamma\right)^{\alpha} G\right\|_{L^{1} L^{2}\left(\mathbb{E}_{+}^{1+3}\right)},
\end{aligned}
$$

if $B$ is a large fixed constant as before. Since $\Gamma^{\alpha} \Psi_{k}=O\left(\operatorname{dist}^{-2|\alpha|}\left(P, P_{0}\right)\right)$ on the support of $F$, we see from the Schwarz inequality that

$$
\sum_{|\alpha| \leq 1}\left\|\left(\operatorname{dist}^{2}\left(P, P_{0}\right) \Gamma\right)^{\alpha}\left(\Psi_{k} F\right)\right\|_{L^{1} L^{2}\left(\mathbb{E}_{+}^{1+3}\right)} \leq C \sum_{|\alpha| \leq 1}\left\|\operatorname{dist}^{1+2|\alpha|}\left(P, P_{0}\right) \Gamma^{\alpha} F\right\|_{L^{2}\left(\mathbb{E}_{+}^{1+3}: T \in I_{k}^{*}\right)},
$$


assuming, as above, that $F$ is supported in $Y_{+}$. By combining the last two inequalities and applying the Schwarz inequality, we get

$$
\begin{aligned}
& \sum_{|\alpha| \leq 1}\left\|\left(\operatorname{dist}^{2}\left(P, P_{0}\right) \Gamma\right)^{\alpha} \mathcal{Q}\right\|_{L^{2}\left(\Lambda_{j}^{+}\right)} \\
& \leq C \sum_{|k-j|<B} \sum_{|\alpha| \leq 1}\left\|\operatorname{dist}^{1+2|\alpha|}\left(P, P_{0}\right) \Gamma^{\alpha} F\right\|_{L^{2}\left(\mathbb{E}_{+}^{1+3}: T \in I_{k}^{*}\right)} \sum_{|\alpha| \leq 1}\left\|\operatorname{dist}^{2|\alpha|} \Gamma^{\alpha} G\right\|_{L^{2}\left(\mathbb{E}_{+}^{1+3}\right)}, \\
& \quad \text { if } \operatorname{support}(F) \subseteq Y_{+} .
\end{aligned}
$$

This implies (5.3). The proof of (5.4) is similar.

We now show that Proposition 5.1 implies the inequality (2.29) where the norm on the left hand side is taken over $\mathbb{E}_{+}^{1+3} \backslash Y_{+}$. Together with the results of Sections 3 and 4 , this will complete the proof of the inequality (2.29). For this, let $\beta$ be the pushforward to $\mathbb{E}_{+}^{1+3}$ of the function $1-\eta(2 x)$. Thus,

$$
\left\{\begin{array}{l}
\beta=1 \text { on } \mathbb{E}_{+}^{1+3} \backslash Y_{+} \\
\beta=0 \text { on a neighborhood of } \mathcal{K}^{*} \\
\Gamma^{\alpha} \beta=O\left(\operatorname{dist}^{-2|\alpha|}\left(P, P_{0}\right)\right) .
\end{array}\right.
$$

We set

$$
u_{f}=\beta u, \quad v_{f}=\beta v,
$$

so that $u_{f}=u$ and $v_{f}=v$ on $Y_{+}^{c}$. Additionally, they solve the free wave equations

$$
\begin{aligned}
& \left(\square_{g}+1\right) u_{f}=\beta F+\left[\square_{g}, \beta\right] u=F_{0}+F_{1} \\
& \left(\square_{g}+1\right) v_{f}=\beta G+\left[\square_{g}, \beta\right] v=G_{0}+G_{1}
\end{aligned}
$$

with vanishing Cauchy data. We then can write $u_{f}=u_{f, 0}+u_{f, 1}$ and $v_{f}=v_{f, 0}+v_{f, 1}$, where the pieces solve the free wave equations

$$
\left(\square_{g}+1\right) u_{f, j}=F_{j}, \quad\left(\square_{g}+1\right) v_{f, j}=G_{j}, \quad j=0,1 .
$$

Note also that

$$
\sum_{|\alpha| \leq 1}\left\|\operatorname{dist}^{2|\alpha|}\left(P, P_{0}\right) \Gamma^{\alpha} F_{0}\right\|_{L^{2}\left(\mathbb{E}_{+}^{1+3}\right)} \leq C \sum_{|\alpha| \leq 1}\left\|\operatorname{dist}^{2|\alpha|}\left(P, P_{0}\right) \Gamma^{\alpha} F\right\|_{L^{2}\left(\mathbb{E}_{+}^{1+3}\right)} .
$$

Furthermore, since $F_{1}$ vanishes on $Y_{+}^{c}$, and

$$
\sum_{|\alpha| \leq 1}\left|\left(\operatorname{dist}^{2}\left(P, P_{0}\right) \Gamma\right)^{\alpha}\left[\square_{g}, \beta\right] u\right| \leq C \sum_{|\alpha| \leq 2} \operatorname{dist}^{2(|\alpha|-2)}\left(P, P_{0}\right)\left|\Gamma^{\alpha} u\right|
$$

Proposition 4.1 yields

$$
\begin{aligned}
\sum_{|\alpha| \leq 1}\left\|\operatorname{dist}^{1+2|\alpha|}\left(P, P_{0}\right) \Gamma^{\alpha} F_{1}\right\|_{L^{2}\left(\mathbb{E}_{+}^{1+3}\right)} \leq & C \sum_{|\alpha| \leq 2}\left\|\operatorname{dist}^{1+2(|\alpha|-2)}\left(P, P_{0}\right) \Gamma^{\alpha} u\right\|_{L^{2}\left(Y_{+}\right)} \\
& \leq C \sum_{|\alpha| \leq 1}\left\|\operatorname{dist}^{2|\alpha|}\left(P, P_{0}\right) \Gamma^{\alpha} F\right\|_{L^{2}\left(\mathbb{E}_{+}^{1+3}\right)} .
\end{aligned}
$$


To proceed, note that (5.2) and (5.5) yield

$$
\begin{aligned}
& \sum_{|\alpha| \leq 1}\left\|\left(\operatorname{dist}^{2}\left(P, P_{0}\right) \Gamma\right)^{\alpha} \mathcal{Q}\left(u_{f, 0}, d u_{f, 0} ; v_{f, 0}, d v_{f, 0}\right)\right\|_{L^{2}\left(\mathbb{E}_{+}^{1+3}\right)} \\
& \quad \leq C \sum_{|\alpha| \leq 1}\left\|\operatorname{dist}^{2|\alpha|}\left(P, P_{0}\right) \Gamma^{\alpha} F\right\|_{L^{2}\left(\mathbb{E}_{+}^{1+3}\right)} \sum_{|\alpha| \leq 1}\left\|\operatorname{dist}^{2|\alpha|}\left(P, P_{0}\right) \Gamma^{\alpha} G\right\|_{L^{2}\left(\mathbb{E}_{+}^{1+3}\right)} .
\end{aligned}
$$

Similarly, (5.3), (5.5) and (5.6) give

$$
\begin{aligned}
& \sum_{|\alpha| \leq 1}\left\|\left(\operatorname{dist}^{2}\left(P, P_{0}\right) \Gamma\right)^{\alpha} \mathcal{Q}\left(u_{f, 1}, d u_{f, 1} ; v_{f, 0}, d v_{f, 0}\right)\right\|_{L^{2}\left(\mathbb{E}_{+}^{1+3}\right)} \\
& \quad+\sum_{\mid \alpha \leq 1}\left\|\left(\operatorname{dist}^{2}\left(P, P_{0}\right) \Gamma\right)^{\alpha} \mathcal{Q}\left(u_{f, 0}, d u_{f, 0} ; v_{f, 1}, d v_{f, 1}\right)\right\|_{L^{2}\left(\mathbb{E}_{+}^{1+3}\right)} \\
& \quad \leq C \sum_{|\alpha| \leq 1}\left\|\operatorname{dist}^{2|\alpha|}\left(P, P_{0}\right) \Gamma^{\alpha} F\right\|_{L^{2}\left(\mathbb{E}_{+}^{1+3}\right)} \sum_{|\alpha| \leq 1}\left\|\operatorname{dist}^{2|\alpha|}\left(P, P_{0}\right) \Gamma^{\alpha} G\right\|_{L^{2}\left(\mathbb{E}_{+}^{1+3}\right)} .
\end{aligned}
$$

Finally, (5.4) and (5.6) imply that

$$
\begin{aligned}
& \sum_{|\alpha| \leq 1}\left\|\left(\operatorname{dist}^{2}\left(P, P_{0}\right) \Gamma\right)^{\alpha} \mathcal{Q}\left(u_{f, 1}, d u_{f, 1} ; v_{f, 1}, d v_{f, 1}\right)\right\|_{L^{2}\left(\mathbb{E}_{+}^{1+3}\right)} \\
& \quad \leq C \sum_{|\alpha| \leq 1}\left\|\operatorname{dist}^{2|\alpha|}\left(P, P_{0}\right) \Gamma^{\alpha} F\right\|_{L^{2}\left(\mathbb{E}_{+}^{1+3}\right)} \sum_{|\alpha| \leq 1}\left\|\operatorname{dist}^{2|\alpha|}\left(P, P_{0}\right) \Gamma^{\alpha} G\right\|_{L^{2}\left(\mathbb{E}_{+}^{1+3}\right)} .
\end{aligned}
$$

If we combine the last three inequalities we conclude that the analog of (2.29) holds if the norm on the left is taken over $Y_{+}^{c}$.

Proof of the estimate (5.1) In what follows, we simplify our notation somewhat by letting

$$
\left|h^{\prime}\right|=\sum_{|\alpha|=1}\left|\Gamma^{\alpha} h\right|, \quad \text { and }\left|h^{\prime \prime}\right|=\sum_{|\alpha|=2}\left|\Gamma^{\alpha} h\right| .
$$

In view of Proposition 2.4, Proposition 5.1 follows as a consequence of the following three lemmas.

Lemma 5.2. Suppose that $Q$ is a standard null form on the cylinder and that $u_{f}$ and $v_{f}$ are solutions of standard inhomogeneous wave equations $\left(\square_{g}+1\right) u_{f}=F$ and $\left(\square_{g}+1\right) v_{f}=$ $G$ with zero initial data. Then

$$
\begin{aligned}
& \sum_{|\alpha| \leq 1}\left\|\operatorname{dist}^{2}\left(P, P_{0}\right)\left(\operatorname{dist}^{2}\left(P, P_{0}\right) \Gamma\right)^{\alpha} Q\left(d u_{f}, d v_{f}\right)\right\|_{L^{2}\left(\mathbb{E}_{+}^{1+3}\right)} \\
& \quad \leq C \sum_{|\alpha| \leq 1}\left\|\operatorname{dist}^{2|\alpha|}\left(P, P_{0}\right) \Gamma^{\alpha} F\right\|_{L^{1} L^{2}\left(\mathbb{E}_{+}^{1+3}\right)}\left\|d i s t^{2|\alpha|}\left(P, P_{0}\right) \Gamma^{\alpha} G\right\|_{L^{1} L^{2}\left(\mathbb{E}_{+}^{1+3}\right)} .
\end{aligned}
$$

Lemma 5.3. Let $u_{f}$ and $v_{f}$ be as above then

$$
\begin{aligned}
& \left\|\operatorname{dist}^{3}\left(P, P_{0}\right) u_{f} v_{f}^{\prime \prime}\right\|_{L^{2}\left(\mathbb{E}_{+}^{1+3}\right)}+\left\|\operatorname{dist}^{3}\left(P, P_{0}\right) u_{f}^{\prime} v_{f}^{\prime}\right\|_{L^{2}\left(\mathbb{E}_{+}^{1+3}\right)} \\
& \quad \leq C \sum_{|\alpha| \leq 1}\left\|\operatorname{dist}^{2|\alpha|}\left(P, P_{0}\right) \Gamma^{\alpha} F\right\|_{L^{1} L^{2}\left(\mathbb{E}_{+}^{1+3}\right)}\left\|\sum_{|\alpha| \leq 1}\right\| \operatorname{dist}^{2|\alpha|}\left(P, P_{0}\right) \Gamma^{\alpha} G \|_{L^{1} L^{2}\left(\mathbb{E}_{+}^{1+3}\right)} .
\end{aligned}
$$


Lemma 5.4. If $u_{f}$ and $v_{f}$ are as above

$$
\begin{aligned}
& \left\|\operatorname{dist}\left(P, P_{0}\right) u_{f}^{\prime} v_{f}\right\|_{L^{2}\left(\mathbb{E}_{+}^{1+3}\right)}+\left\|u_{f} v_{f}\right\|_{L^{2}\left(\mathbb{E}_{+}^{1+3}\right)} \\
& \quad \leq C \sum_{|\alpha| \leq 1}\left\|\operatorname{dist} t^{2|\alpha|}\left(P, P_{0}\right) \Gamma^{\alpha} F\right\|_{L^{1} L^{2}\left(\mathbb{E}_{+}^{1+3}\right)} \sum_{|\alpha| \leq 1}\left\|d i s t^{2|\alpha|}\left(P, P_{0}\right) \Gamma^{\alpha} G\right\|_{L^{1} L^{2}\left(\mathbb{E}_{+}^{1+3}\right)} .
\end{aligned}
$$

Proof of Lemma 5.2 We apply an estimate from [20], which says that

$$
\sum_{|\alpha| \leq 1}\left\|\Gamma^{\alpha} Q\left(d u_{f}, d v_{f}\right)\right\|_{L^{2}\left(\mathbb{E}_{+}^{1+3}\right)} \leq C \sum_{|\alpha| \leq 1}\left\|\Gamma^{\alpha} F\right\|_{L^{1} L^{2}\left(\mathbb{E}_{+}^{1+3}\right)} \sum_{|\alpha| \leq 1}\left\|\Gamma^{\alpha} G\right\|_{L^{1} L^{2}\left(\mathbb{E}_{+}^{1+3}\right)} .
$$

Next, we fix a partition of unity $\sum \beta\left(2^{j} s\right)=1, s>0$ with $\operatorname{supp} \beta \subset[1 / 2,2]$, and let $F_{j}=\beta\left(2^{j} \operatorname{dist}\left(P, P_{0}\right)\right) F$. From the preceeding estimate, Huygen's principle, and the Schwarz inequality, one sees that for some fixed $B$, the following holds for $k=0,1,2, \ldots$

$$
\begin{aligned}
& \sum_{|\alpha|=1}\left\|\operatorname{dist}^{4}\left(P, P_{0}\right) \Gamma^{\alpha} Q\right\|_{L^{2}\left(\operatorname{dist}\left(P, P_{0}\right) \approx 2^{-k}\right)} \\
& \leq C \sum_{|\alpha| \leq 1} \sum_{j \leq k+B}\left\|2^{-2 k} \Gamma^{\alpha} F_{j}\right\|_{L^{1} L^{2}} \sum_{|\alpha| \leq 1} \sum_{j \leq k+B}\left\|2^{-2 k} \Gamma^{\alpha} G_{j}\right\|_{L^{1} L^{2}} \\
& \leq C \sum_{|\alpha| \leq 1} \sum_{j \leq k+B} 2^{-2(k-j)}\left\|\operatorname{dist}^{2|\alpha|}\left(P, P_{0}\right) \Gamma^{\alpha} F\right\|_{L^{1} L^{2}\left(\operatorname{dist}\left(P, P_{0}\right) \approx 2^{-j}\right)} \\
& \times \sum_{|\alpha| \leq 1} \sum_{j \leq k+B} 2^{-2(k-j)}\left\|\operatorname{dist}^{2|\alpha|}\left(P, P_{0}\right) \Gamma^{\alpha} G\right\|_{L^{1} L^{2}\left(\operatorname{dist}\left(P, P_{0}\right) \approx 2^{-j}\right)} .
\end{aligned}
$$

This yields the estimate for the $|\alpha|=1$ terms on the left side of (5.7).

To estimate the term with $\alpha=0$ on the left side of (5.7), we let $u_{f, k}$ be the solution of the inhomogeneous wave equation $\left(\square_{g}+1\right) u_{f, k}=F_{k}$. We then can write

$$
Q=\sum_{j \leq l} Q\left(d u_{f, j}, d v_{f, l}\right)+\sum_{j>l} Q\left(d u_{f, k}, d v_{f, l}\right) .
$$

Since the two terms are similar, we shall only estimate the first one. We use the following estimate from 20].

$$
\|Q\|_{L^{2}\left(\mathbb{E}_{+}^{1+3}\right)} \leq C \sum_{|\alpha| \leq 1}\left\|\Gamma^{\alpha} F\right\|_{L^{1} L^{2}\left(\mathbb{E}_{+}^{1+3}\right)}\|G\|_{L^{1} L^{2}\left(\mathbb{E}_{+}^{1+3}\right)},
$$

We apply this to obtain

$$
\begin{gathered}
\left\|\operatorname{dist}^{2}\left(P, P_{0}\right) \sum_{j \leq l} Q\left(d u_{f, j}, d v_{f, l}\right)\right\|_{L^{2}\left(\operatorname{dist}\left(P, P_{0}\right) \approx 2^{-k}\right)} \leq C \sum_{|\alpha| \leq 1} \sum_{j \leq l \leq k+B} 2^{-2 k}\left\|\Gamma^{\alpha} F_{j}\right\|_{L^{1} L^{2}}\left\|G_{l}\right\|_{L^{1} L^{2}} \\
\leq C \sum_{|\alpha| \leq 1} \sum_{j \leq l \leq k+B} 2^{-2(k-j)}\left\|\operatorname{dist}^{2|\alpha|}\left(P, P_{0}\right) \Gamma^{\alpha} F\right\|_{L^{1} L^{2}\left(\operatorname{dist}\left(P, P_{0}\right) \approx 2^{-j}\right)}\|G\|_{L^{1} L^{2}\left(\operatorname{dist}\left(P, P_{0}\right) \approx 2^{-l}\right)} \\
\leq C \sum_{|\alpha| \leq 1} \sum_{j \leq k+B} 2^{-(k-j)}\left\|\operatorname{dist}^{2|\alpha|}\left(P, P_{0}\right) \Gamma^{\alpha} F\right\|_{L^{1} L^{2}\left(\operatorname{dist}\left(P, P_{0}\right) \approx 2^{-j}\right)} \\
\times \sum_{l \leq k+B} 2^{-(k-l)}\|G\|_{L^{1} L^{2}\left(\operatorname{dist}\left(P, P_{0}\right) \approx 2^{-l}\right)}
\end{gathered}
$$


This yields the desired estimate for the $|\alpha|=0$ term in the left of (5.7), completing the proof of Lemma 5.2.

In the proof of Lemma 5.3, we shall need to make use of the following estimate.

Lemma 5.5. Let $B_{\varepsilon}$ denote a spherical cap of radius $\varepsilon>0$ in $S^{3}$. Then

$$
\|h\|_{L^{\infty}\left(B_{\varepsilon}\right)} \leq C \varepsilon^{1 / 2}\left\|h^{\prime}\right\|_{L^{6}\left(B_{\varepsilon}\right)}+C \varepsilon^{-1 / 2}\|h\|_{L^{6}\left(B_{\varepsilon}\right)} .
$$

To prove this one first notices that it follows from the Euclidean version, which in turn follows from the $\varepsilon=1$ case and a simple scaling argument.

Proof of Lemma 5.3. We start by estimating the first term on the left hand side of (5.8) since it is the more difficult. We use the preceeding lemma to obtain

$$
\begin{aligned}
\left\|u_{f}\right\|_{L^{\infty}\left(\operatorname{dist}\left(P, P_{0}\right) \approx 2^{-k}\right)} & \leq \sum_{j \leq k+B}\left\|u_{f, j}\right\|_{L^{\infty}\left(\operatorname{dist}\left(P, P_{0}\right) \leq 2^{-k}\right)} \\
& \leq \sum_{j \leq k+B} 2^{-k / 2}\left\|u_{f, j}^{\prime}\right\|_{L^{\infty} L^{6}}+2^{k / 2}\left\|u_{f, j}\right\|_{L^{\infty} L^{6}} \\
& \leq \sum_{j \leq k+B} 2^{-k / 2}\left\|F_{j}^{\prime}\right\|_{L^{1} L^{2}}+2^{k / 2}\left\|F_{j}\right\|_{L^{1} L^{2}} \\
& \leq \sum_{j \leq k+B} 2^{-k / 2}\|F\|_{L^{1} L^{2}\left(\operatorname{dist}\left(P, P_{0}\right) \approx 2^{-j}\right)}+2^{k / 2}\|F\|_{L^{1} L^{2}\left(\operatorname{dist}\left(P, P_{0}\right) \approx 2^{-j}\right)}
\end{aligned}
$$

As a result, for fixed $k=0,1,2, \ldots$,

$$
\begin{aligned}
&\left\|\operatorname{dist}^{3}\left(P, P_{0}\right) u_{f} v_{f}^{\prime \prime}\right\|_{L^{2}\left(\operatorname{dist}\left(P, P_{0}\right) \approx 2^{-k}\right)} \\
& \leq C 2^{-3 k-k / 2}\left\|v_{f}^{\prime \prime}\right\|_{L^{\infty} L^{2}\left(\operatorname{dist}\left(P, P_{0}\right) \approx 2^{-k}\right)}\left\|u_{f}\right\|_{L^{\infty}\left(\operatorname{dist}\left(P, P_{0}\right) \approx 2^{-k}\right)} \\
& \leq C 2^{-2 k} \sum_{j \leq k+B}\left\|G^{\prime}\right\|_{L^{1} L^{2}\left(\operatorname{dist}\left(P, P_{0}\right) \approx 2^{-j}\right)} \\
& \times\left(2^{-2 k} \sum_{j \leq k+B}\left\|F^{\prime}\right\|_{L^{1} L^{2}\left(\operatorname{dist}\left(P, P_{0}\right) \approx 2^{-j}\right)}+2^{-k} \sum_{j \leq k+B}\|F\|_{L^{1} L^{2}\left(\operatorname{dist}\left(P, P_{0}\right) \approx 2^{-j}\right)}\right) \\
& \leq C \sum_{j \leq k+B} 2^{-2(k-j)}\left\|\operatorname{dist}^{2}\left(P, P_{0}\right) G^{\prime}\right\|_{L^{1} L^{2}\left(\operatorname{dist}\left(P, P_{0}\right) \approx 2^{-j}\right)} \\
& \quad \times \sum_{j \leq k+B}\left(2^{-2(k-j)}\left\|\operatorname{dist}^{2}\left(P, P_{0}\right) F^{\prime}\right\|_{L^{1} L^{2}\left(\operatorname{dist}\left(P, P_{0}\right) \approx 2^{-j}\right)}+2^{-k}\|F\|_{L^{1} L^{2}\left(\operatorname{dist}\left(P, P_{0}\right) \approx 2^{-j}\right)}\right) .
\end{aligned}
$$

This implies that the first term on the left side of (5.8) satisfies the stated estimate.

For the second term in (5.8), we first use Hölder's inequality to obtain

$$
\begin{aligned}
& \left\|\operatorname{dist}^{3}\left(P, P_{0}\right) u_{f}^{\prime} v_{f}^{\prime}\right\|_{L^{2}\left(\operatorname{dist}\left(P, P_{0}\right) \approx 2^{-k}\right)} \\
& \quad \leq C 2^{-4 k}\left\|u_{f}^{\prime}\right\|_{L^{\infty} L^{6}\left(\operatorname{dist}\left(P, P_{0}\right) \approx 2^{-k}\right)}\left\|v_{f}^{\prime}\right\|_{L^{\infty} L^{6}\left(\operatorname{dist}\left(P, P_{0}\right) \approx 2^{-k}\right)}
\end{aligned}
$$

As above, we can bound

$$
\begin{aligned}
& 2^{-2 k}\left\|u_{f}^{\prime}\right\|_{L^{\infty} L^{6}\left(\operatorname{dist}\left(P, P_{0}\right) \approx 2^{-k}\right)} \\
\leq & \sum_{j \leq k+B}\left(2^{-2(k-j)}\left\|\operatorname{dist}^{2}\left(P, P_{0}\right) F^{\prime}\right\|_{L^{1} L^{2}\left(\operatorname{dist}\left(P, P_{0}\right) \approx 2^{-j}\right)}+2^{-k}\|F\|_{L^{1} L^{2}\left(\operatorname{dist}\left(P, P_{0}\right) \approx 2^{-j}\right)}\right)
\end{aligned}
$$


which leads to the desired bounds for the remaining term, completing the proof of Lemma 5.3 .

Proof of Lemma 5.4 Let $u_{f, j}$ and $v_{f, l}$ be as in the proof of Lemma 5.2. We then can write

$$
u_{f}^{\prime} v_{f}=\sum_{j \leq l} u_{f, j}^{\prime} v_{f, l}+\sum_{j>l} u_{f, j}^{\prime} v_{f, l} .
$$

To handle the terms with $j \leq l$, we note that Hölder's inequality and the above arguments yield

$$
\begin{aligned}
& \sum_{j \leq l \leq k+B}\left\|\operatorname{dist}\left(P, P_{0}\right) u_{f, j}^{\prime} v_{f, l}\right\|_{L^{2}\left(\operatorname{dist}\left(P, P_{0}\right) \approx 2^{-k}\right)} \\
& \leq C 2^{-2 k} \sum_{j \leq l \leq k+B}\left\|u_{f, j}^{\prime}\right\|_{L^{\infty} L^{6}}\left\|v_{f, l}\right\|_{L^{\infty} L^{6}} \\
& \leq C \sum_{j \leq l \leq k+B}\left(2^{-2(k-j)}\left\|\operatorname{dist}^{2}\left(P, P_{0}\right) F^{\prime}\right\|_{L^{1} L^{2}\left(\operatorname{dist}\left(P, P_{0}\right) \approx 2^{-j}\right)}+2^{-k}\|F\|_{L^{1} L^{2}}\right) \\
& \times\|G\|_{L^{1} L^{2}\left(\operatorname{dist}\left(P, P_{0}\right) \approx 2^{-l}\right)} .
\end{aligned}
$$

Since $k-j \geq k-l$, this implies the estimate for these terms. To handle the terms where $j>l$ we write

$$
\begin{aligned}
& \sum_{l \leq j \leq k+B}\left\|\operatorname{dist}\left(P, P_{0}\right) u_{f, j}^{\prime} v_{f, l}\right\|_{L^{2}\left(\operatorname{dist}\left(P, P_{0}\right) \approx 2^{-k}\right)} \\
& \leq C 2^{-3 k / 2} \sum_{l \leq j \leq k+B}\left\|u_{f, j}^{\prime}\right\|_{L^{\infty} L^{2}\left(\operatorname{dist}\left(P, P_{0}\right) \approx 2^{-k}\right)}\left\|v_{f, l}\right\|_{L^{\infty}\left(\operatorname{dist}\left(P, P_{0}\right) \leq 2^{k}\right)} \\
& \leq C \sum_{l \leq j \leq k+B}\|F\|_{L^{1} L^{2}\left(\operatorname{dist}\left(P, P_{0}\right) \approx 2^{-j}\right)} \\
& \quad \times\left(2^{-2(k-l)}\left\|\operatorname{dist}\left(P, P_{0}\right)^{2} G^{\prime}\right\|_{L^{1} L^{2}\left(\operatorname{dist}\left(P, P_{0}\right) \approx 2^{-l}\right)}+2^{-k}\|G\|_{L^{1} L^{2}}\right) .
\end{aligned}
$$

This establishes the desired estimate for the terms with $j>l$, which along with the preceding estimate shows that the first term on the left side of (5.9) satisfies the desired bounds.

To handle the second term on the left hand side of (5.9), we apply Hölder's inequality to deduce that

$$
\begin{aligned}
\left\|u_{f} v_{f}\right\|_{L^{2}\left(\operatorname{dist}\left(P, P_{0}\right) \approx 2^{-k}\right)} & \leq C 2^{-k}\left\|u_{f}\right\|_{L^{\infty} L^{6}\left(\operatorname{dist}\left(P, P_{0}\right) \approx 2^{-k}\right)}\left\|v_{f}\right\|_{L^{\infty} L^{6}\left(\operatorname{dist}\left(P, P_{0}\right) \approx 2^{-k}\right)} \\
& \leq C 2^{-k}\|F\|_{L^{1} L^{2}\left(\operatorname{dist}\left(P, P_{0}\right) \geq 2^{-k+B}\right)}\|G\|_{L^{1} L^{2}\left(\operatorname{dist}\left(P, P_{0}\right) \geq 2^{-k+B}\right)}
\end{aligned}
$$

which implies the desired estimate, completing the proof of Lemma 5.4.

\section{End of Proof of Theorem 2.3: Weighted Pecher Estimates.}

We still need to prove (2.30), which is the weighted Pecher inequality

$$
\begin{aligned}
& \sum_{|\alpha| \leq 1}\left\|\left(\operatorname{dist}^{2}\left(P, P_{0}\right) \Gamma\right)^{\alpha} u\right\|_{L^{8}\left(\mathbb{E}_{+}^{1+3} \backslash \mathcal{K}_{*}\right)} \\
& \quad \leq C\left(\left\|u_{0}\right\|_{H_{D}^{2}}+\left\|u_{1}\right\|_{H_{D}^{1}}+\sum_{|\alpha| \leq 1}\left\|\left(\operatorname{dist}^{2}\left(P, P_{0}\right) \Gamma\right)^{\alpha} F\right\|_{L^{2}\left(\mathbb{E}_{+}^{1+3} \backslash \mathcal{K}_{*}\right)}\right) .
\end{aligned}
$$


The local version of the Pecher inequalities for variable coefficient wave equations was established in [14]. In particular, that result implies the following result for the free wave equation on the Einstein cylinder

$$
\left\|u_{f}\right\|_{L^{8}\left(\mathbb{E}_{+}^{1+3}\right)} \leq C\left(\left\|u_{f}(0, \cdot)\right\|_{H^{1}}+\left\|\partial_{T} u_{f}(0, \cdot)\right\|_{L^{2}}+\|F\|_{L^{1} L^{2}\left(\mathbb{E}_{+}^{1+3}\right)}\right) .
$$

Also, the local version of the Pecher inequalities for the obstacle problem in Minkowski space were established by the authors in [17],

$$
\|\tilde{u}\|_{L^{8}\left([0,1] \times \mathbb{R}^{3} \backslash \mathcal{K}\right)} \leq C\left(\left\|\tilde{u}_{0}\right\|_{H_{D}^{1}}+\left\|\tilde{u}_{1}\right\|_{L^{2}}+\|\tilde{F}\|_{L^{1} L^{2}\left([0,1] \times \mathbb{R}^{3} \backslash \mathcal{K}\right)}\right) .
$$

We also need the version with first order derivatives of $\tilde{u}$, which follows from the above and an integration by parts argument in $t$,

$$
\sum_{|\alpha| \leq 1}\left\|D^{\alpha} \tilde{u}\right\|_{L^{8}\left([0,1] \times \mathbb{R}^{3} \backslash \mathcal{K}\right)} \leq C\left(\left\|\tilde{u}_{0}\right\|_{H_{D}^{2}}+\left\|\tilde{u}_{1}\right\|_{H_{D}^{1}}+\sum_{|\alpha| \leq 1}\left\|D^{\alpha} \tilde{F}\right\|_{L^{1} L^{2}\left([0,1] \times \mathbb{R}^{3} \backslash \mathcal{K}\right)}\right) .
$$

Together, (6.2) and (6.3) allow one to reduce the proof of the estimate (6.1) to the case that $u_{0}=u_{1}=0$, following the arguments at the end of the second section of this paper. The proof of (6.1) now follows very closely the proof of (2.29). As in the proof of that estimate, the first step is to control the norm over $Y_{+}$. To do this, we note that

$$
\sum_{|\alpha| \leq 1}\left\|\left(\operatorname{dist}^{2}\left(P, P_{0}\right) \Gamma\right)^{\alpha} u\right\|_{L^{8}\left(Y_{+}\right)} \approx \sum_{|\alpha| \leq 1}\left\|(1+t) D^{\alpha} \tilde{u}\right\|_{L^{8}(|x| \leq A, t>0)} .
$$

Next, if $\square \tilde{u}^{1}=\tilde{F}^{1}$, with vanishing Cauchy data, and $\tilde{F}^{1}$ is supported in the set $|x| \leq 2 A$, then energy decay and the local estimates $(6.3)$ imply the following

$$
\sum_{|\alpha| \leq 1}\left\|(1+t) D^{\alpha} \tilde{u}^{1}\right\|_{L^{8}(|x| \leq A, t>0)} \leq \sum_{|\alpha| \leq 1}\left\|(1+t) D^{\alpha} \tilde{F}^{1}\right\|_{L^{2}(d x d t)} .
$$

If we set $\tilde{u}^{1}=\eta \tilde{u}$, then (see (3.3), (3.4), and Proposition 4.1) we obtain the estimate

$$
\begin{aligned}
& \sum_{|\alpha| \leq 1}\left\|\left(\operatorname{dist}^{2}\left(P, P_{0}\right) \Gamma\right)^{\alpha} u\right\|_{L^{8}\left(Y_{+}\right)} \\
& \leq C\left(\left\|u_{0}\right\|_{H_{D}^{2}}+\left\|u_{1}\right\|_{H_{D}^{1}}+\sum_{|\alpha| \leq 1}\left\|\left(\operatorname{dist}^{2}\left(P, P_{0}\right) \Gamma\right)^{\alpha} F\right\|_{L^{2}\left(\mathbb{E}_{+}^{1+3} \backslash \mathcal{K}_{*}\right)}\right) .
\end{aligned}
$$

To handle the norm over the complement of $Y_{+}$, we will use the following estimates for the free wave equation on $\mathbb{E}_{+}^{1+3}$,

$$
\sum_{|\alpha| \leq 1}\left\|\left(\operatorname{dist}^{2}\left(P, P_{0}\right) \Gamma\right)^{\alpha} u_{f}\right\|_{L^{8}\left(\mathbb{E}_{+}^{1+3}\right)} \leq C \sum_{|\alpha| \leq 1}\left\|\operatorname{dist}^{2|\alpha|}\left(P, P_{0}\right) \Gamma^{\alpha} F\right\|_{L^{2}\left(\mathbb{E}_{+}^{1+3}\right)}
$$

and the improved estimate for data supported near the boundary

$$
\begin{aligned}
\sum_{|\alpha| \leq 1}\left\|\left(\operatorname{dist}^{2}\left(P, P_{0}\right) \Gamma\right)^{\alpha} u_{f}\right\|_{L^{8}\left(\mathbb{E}_{+}^{1+3}\right)} \leq C \sum_{|\alpha| \leq 1}\left\|\operatorname{dist}^{1+2|\alpha|}\left(P, P_{0}\right) \Gamma^{\alpha} F\right\|_{L^{2}\left(\mathbb{E}_{+}^{1+3}\right)} \\
\quad \text { if } \operatorname{support}(F) \subseteq Y_{+} .
\end{aligned}
$$


The estimate (6.5) is a consequence of (6.4) by the same steps as (5.3) follows from (5.1). And, by letting $u_{f}=\beta u$, the following is a consequence of (6.4) and (6.5)

$$
\begin{aligned}
& \sum_{|\alpha| \leq 1}\left\|\left(\operatorname{dist}^{2}\left(P, P_{0}\right) \Gamma\right)^{\alpha} u\right\|_{L^{8}\left(\mathbb{E}_{+}^{1+3} \backslash Y_{+}\right)} \\
& \leq C\left(\left\|u_{0}\right\|_{H_{D}^{2}}+\left\|u_{1}\right\|_{H_{D}^{1}}+\sum_{|\alpha| \leq 1}\left\|\left(\operatorname{dist}^{2}\left(P, P_{0}\right) \Gamma\right)^{\alpha} F\right\|_{L^{2}\left(\mathbb{E}_{+}^{1+3} \backslash \mathcal{K}_{*}\right)}\right) .
\end{aligned}
$$

It thus remains only to establish the estimate (6.4). For the $|\alpha|=0$ terms, this is just the estimate (6.2). Next, from the fact that $\Gamma^{\alpha}$ commutes with $\square_{g}$, we obtain the following

$$
\sum_{|\alpha|=1}\left\|\Gamma^{\alpha} u_{f}\right\|_{L^{8}\left(\operatorname{dist}\left(P, P_{0}\right) \approx 2^{-j}\right)} \leq C \sum_{|\alpha|=1}\left\|\Gamma^{\alpha} F\right\|_{L^{2}\left(\operatorname{dist}\left(P, P_{0}\right) \geq 2^{-j+B}\right)}
$$

for $B$ fixed as before. Summing over $j$ yields (6.4).

We conclude this section with a simple corollary of our weighted Pecher estimate (6.1). We first see that, if $u$ is as above, then

$$
(\pi-T)|u(T, \cdot)| \leq C\left(\left\|u_{0}\right\|_{H_{D}^{2}}+\left\|u_{1}\right\|_{H_{D}^{1}}+\sum_{|\alpha| \leq 1}\left\|\left(\operatorname{dist}^{2}\left(P, P_{0}\right) \Gamma\right)^{\alpha} F\right\|_{L^{2}\left(\mathbb{E}_{+}^{1+3} \backslash \mathcal{K}_{*}\right)}\right) .
$$

To prove this, one uses the fact that, if $B_{\varepsilon}$ is a ball of radius $\varepsilon>0$, then

$$
\|h\|_{L^{\infty}\left(B_{\varepsilon}\right)} \leq C \varepsilon^{1 / 2} \sum_{|\alpha|=1}\left\|\Gamma^{\alpha} h\right\|_{L^{8}\left(B_{\varepsilon}\right)}+C \varepsilon^{-1 / 2}\|h\|_{L^{8}\left(B_{\varepsilon}\right)},
$$

which follows from Euclidean estimates. Given $(T, X) \in \overline{\mathbb{E}}_{+}^{1+3} \backslash \mathcal{K}_{*}$, we take $\varepsilon=(\pi-T)^{2}$, and $B_{\varepsilon}$ to be a ball of radius $\varepsilon$ such that $\left(T, B_{\varepsilon}\right)$ is contained in $\overline{\mathbb{E}}_{+}^{1+3} \backslash \mathcal{K}_{*}$. We then obtain from (6.1) the following inequality,

$$
\begin{aligned}
|u(T, X)| & \leq C(\pi-T) \sum_{|\alpha|=1}\left\|\Gamma^{\alpha} u\right\|_{L^{8}\left(B_{\varepsilon}\right)}+C(\pi-T)^{-1}\|u\|_{L^{8}\left(B_{\varepsilon}\right)} \\
& \leq C(\pi-T)^{-1} \sum_{|\alpha| \leq 1}\left\|\operatorname{dist}^{2|\alpha|}\left(P, P_{0}\right) \Gamma^{\alpha} u\right\|_{L^{8}\left(\overline{\mathbb{E}}_{+}^{1+3} \backslash \mathcal{K}_{*}\right)} \\
& \leq C(\pi-T)^{-1}\left(\left\|u_{0}\right\|_{H_{D}^{2}}+\left\|u_{1}\right\|_{H_{D}^{1}}+\sum_{|\alpha| \leq 1}\left\|\left(\operatorname{dist}^{2}\left(P, P_{0}\right) \Gamma\right)^{\alpha} F\right\|_{L^{2}\left(\mathbb{E}_{+}^{1+3} \backslash \mathcal{K}_{*}\right)}\right)
\end{aligned}
$$

as claimed.

We now conclude that the solution to equation (1.3) decays like $1 / t$. For this we note that, if $u$ and $\tilde{u}=\mathcal{P}^{*}(\Omega u)$ are identified as before, and $\mathcal{P}(t, x)=(T, X)$, then

$$
\begin{aligned}
|\tilde{u}(t, x)|= & |\Omega u(T, X)| \\
& \leq C t^{-1}\left(\left\|u_{0}\right\|_{H_{D}^{2}}+\left\|u_{1}\right\|_{H_{D}^{1}}+\sum_{|\alpha| \leq 1}\left\|\left(\operatorname{dist}^{2}\left(P, P_{0}\right) \Gamma\right)^{\alpha} F\right\|_{L^{2}\left(\mathbb{E}_{+}^{1+3} \backslash \mathcal{K}_{*}\right)}\right) .
\end{aligned}
$$

This inequality uses the fact that in $\overline{\mathbb{E}}_{+}^{1+3} \backslash \mathcal{K}_{*}$, we have $|\Omega /(\pi-T)| \leq C / t$. 


\section{Global Existence of $H^{2}$ Solutions: Proof of Theorem 1.1.}

Recall that we need to show that if the Cauchy data satisfies

$$
\|f\|_{H_{D}^{2,1}\left(\mathbb{R}^{3} \backslash \mathcal{K}\right)}+\|g\|_{H_{D}^{1,2}\left(\mathbb{R}^{3} \backslash \mathcal{K}\right)}<\varepsilon_{0},
$$

with $\varepsilon_{0}>0$ small, then the equation

$$
\left\{\begin{array}{l}
\square u(t, x)=Q(d u(t, x), d u(t, x)), \quad(t, x) \in \mathbb{R}_{+} \times \mathbb{R}^{3} \backslash \mathcal{K} \\
\left.u\right|_{\partial \mathcal{K}}=0 \\
\left.u\right|_{t=0}=f,\left.\quad \partial_{t} u\right|_{t=0}=g
\end{array}\right.
$$

has a unique global solution verifying $(1.9)$ and (1.10). Note that implicit in (7.1) is that the data satisfy the $H^{2}$ compatibility conditions that both $f$ and $g$ vanish on $\partial \mathcal{K}$.

To avoid cumbersome notation we are switching our notation from the last several sections. In this section and the one to follow we do not denote functions and derivatives on Minkowski space with a tilde.

The uniqueness assertion follows immediately from (1.17). As we shall see, the existence assertion follows easily from Theorem 2.3. Precisely, we shall use Theorem 2.3 to solve the corresponding equation on the Einstein cylinder minus the obstacle. Restricting this solution to the Einstein diamond yields a solution to (7.2), after pulling back via the Penrose transform. Thus, let

$$
u=\Omega \mathcal{P}^{*} v
$$

denote $\Omega$ times the pullback of $v$ via the Penrose map. (In our previous notation $u$ would be $\tilde{v}$.) Then, as noted before, (7.2) is implied by the following

$$
\left\{\begin{array}{l}
\left(\square_{g}+1\right) v(T, X)=\mathcal{Q}(v(T, X), d v(T, X) ; v(T, X), d v(T, X)), \quad(T, X) \in \mathbb{E}_{+}^{1+3} \backslash \mathcal{K}_{*} \\
v(T, X)=0, \quad(T, X) \in \mathcal{K}_{*} \\
\left.v\right|_{T=0}=f_{e},\left.\quad \partial_{T} v\right|_{T=0}=g_{e},
\end{array}\right.
$$

assuming that $f=\Omega \mathcal{P}_{0}^{*} f_{e}$ and $g=\Omega^{2} \mathcal{P}_{0}^{*} g_{e}$, with $\mathcal{P}_{0}$ denoting the restriction of the Penrose map to $t=0$.

To construct a solution of (7.3) on the Einstein cylinder, we let $v$ denote the solution of the following linear equation

$$
\left\{\begin{array}{l}
\left(\square_{g}+1\right) v=F, \quad(T, X) \in \mathbb{E}_{+}^{1+3} \backslash \mathcal{K}_{*} \\
\left.v\right|_{\partial \mathcal{K}_{*}}=0 \\
\left.v\right|_{T=0}=f_{e},\left.\quad \partial_{T} v\right|_{T=0}=g_{e}
\end{array}\right.
$$

The existence of a solution to this linear equation on the Einstein diamond minus the obstacle is obtained from the corresponding solution on Minkowski space. That solution is then easily extended to the Einstein cylinder minus the obstacle. Now let

$$
\mathcal{T} F=\mathcal{Q}(v, d v ; v, d v),
$$

where $v$ solves the above linear equation. Finding a solution to $(7.3)$ is thus reduced to finding a fixed point for the operator $\mathcal{T}$ on the set of $F$ such that

$$
\sum_{|\alpha| \leq 1}\left\|\left(\operatorname{dist}^{2}\left(P, P_{0}\right) \Gamma\right)^{\alpha} F\right\|_{L^{2}\left(\mathbb{E}_{+}^{1+3} \backslash \mathcal{K}_{*}\right)} \leq c_{0} .
$$


We next observe that, by Theorem 2.3,

$$
\sum_{|\alpha| \leq 1}\left\|\left(\operatorname{dist}^{2}\left(P, P_{0}\right) \Gamma\right)^{\alpha} \mathcal{T}(0)\right\|_{L^{2}\left(\mathbb{E}_{+}^{1+3} \backslash \mathcal{K}_{*}\right)} \leq C \varepsilon_{0},
$$

and

$$
\begin{aligned}
& \sum_{|\alpha| \leq 1}\left\|\left(\operatorname{dist}^{2}\left(P, P_{0}\right) \Gamma\right)^{\alpha}\left(\mathcal{T} F_{1}-\mathcal{T} F_{2}\right)\right\|_{L^{2}\left(\mathbb{E}_{+}^{1+3} \backslash \mathcal{K}_{*}\right)} \\
& \leq \sum_{|\alpha| \leq 1}\left\|\left(\operatorname{dist}^{2}\left(P, P_{0}\right) \Gamma\right)^{\alpha} \mathcal{Q}\left(v_{1}-v_{2}, d\left(v_{1}-v_{2}\right) ; v_{1}, d v_{2}\right)\right\|_{L^{2}\left(\mathbb{E}_{+}^{1+3} \backslash \mathcal{K}_{*}\right)} \\
& \quad+\sum_{|\alpha| \leq 1}\left\|\left(\operatorname{dist}^{2}\left(P, P_{0}\right) \Gamma\right)^{\alpha} \mathcal{Q}\left(v_{2}, d v_{2} ; v_{1}-v_{2}, d\left(v_{1}-v_{2}\right)\right)\right\|_{L^{2}\left(\mathbb{E}_{+}^{1+3} \backslash \mathcal{K}_{*}\right)} \\
& \quad \leq 2 C\left(c_{0}+\varepsilon_{0}\right) \cdot \sum_{|\alpha| \leq 1}\left\|\left(\operatorname{dist}^{2}\left(P, P_{0}\right) \Gamma\right)^{\alpha}\left(F_{1}-F_{2}\right)\right\|_{L^{2}\left(\mathbb{E}_{+}^{1+3} \backslash \mathcal{K}_{*}\right)} .
\end{aligned}
$$

Taking $c_{0}=2 C \varepsilon_{0}$, then, for $\varepsilon_{0}>0$ small enough, the contraction principle yields a fixed point for $\mathcal{T}$, hence a solution to $(7.3)$. The corresponding function $u$ defined as above by $u=\Omega \mathcal{P}_{*} v$ is then a solution of $(7.2)$, and it must verify (1.9) by appealing to (1.17), and also must satisfy the decay estimate (1.10) because of $(6.6)$.

8. Global Existence of Smooth Solutions: Proof of Theorem 1.3. To establish Theorem 1.3, we will show that, if the data $f$ and $g$ are smooth and satisfy the appropriate compatibility conditions to infinite order, then the solution $u$ given by Theorem 1.1 belongs to $C^{\infty}\left(\mathbb{R}_{+} \times \mathbb{R}^{3} \backslash \mathcal{K}\right)$. The proof is based on the following Lemma.

Lemma 8.1. Let $u \in C^{j}\left([0, T] ; H_{D}^{2-j}\left(\mathbb{R}^{3} \backslash \mathcal{K}\right)\right), j=0,1,2$, be a solution to (1.3). Then if $v \in C^{j}\left([0, T] ; H_{D}^{1-j}\left(\mathbb{R}^{3} \backslash \mathcal{K}\right)\right), j=0,1$, solves

$$
\left\{\begin{array}{l}
\square v=Q(d u, d v)+Q(d v, d u)+F, \quad(t, x) \in \mathbb{R}_{+} \times \mathbb{R}^{3} \backslash \mathcal{K} \\
\left.v(t, \cdot)\right|_{\partial \mathcal{K}}=0 \\
v(0, \cdot) \in H_{D}^{2}\left(\mathbb{R}^{3} \backslash \mathcal{K}\right), \partial_{t} v(0, \cdot) \in H_{D}^{1}\left(\mathbb{R}^{3} \backslash \mathcal{K}\right),
\end{array}\right.
$$

where $F, D F \in L^{1}\left([0, T] ; L^{2}\left(\mathbb{R}^{3} \backslash \mathcal{K}\right)\right)$, and the following a priori assumption holds,

$$
Q(d u, d v), Q(d v, d u) \in L^{1}\left([0, T] ; L^{2}\left(\mathbb{R}^{3} \backslash \mathcal{K}\right)\right),
$$

then $v \in C^{j}\left([0, T] ; H_{D}^{2-j}\left(\mathbb{R}^{3} \backslash \mathcal{K}\right)\right), j=0,1,2$, and

$$
D Q(d u, d v), D Q(d v, d u) \in L^{1}\left([0, T] ; L^{2}\left(\mathbb{R}^{3} \backslash \mathcal{K}\right)\right) .
$$

Proof. We use the following estimates from [18], for solutions $u, v$ to the CauchyDirichlet problem,

$$
\begin{aligned}
& \|D Q(d u, d v)\|_{L^{2}\left([0, T] \times \mathbb{R}^{3} \backslash \mathcal{K}\right)} \\
& \leq C(u, T)\left(\left\|v_{0}\right\|_{H_{D}^{2}\left(\mathbb{R}^{3} \backslash \mathcal{K}\right)}+\left\|v_{1}\right\|_{H_{D}^{1}\left(\mathbb{R}^{3} \backslash \mathcal{K}\right)}+\sum_{|\alpha| \leq 1} \int_{0}^{T}\left\|D^{\alpha} \square v(s, \cdot)\right\|_{L^{2}\left(\mathbb{R}^{3} \backslash \mathcal{K}\right)} d s\right),
\end{aligned}
$$




$$
\begin{aligned}
& \|Q(d u, d v)\|_{L^{2}\left([0, T] \times \mathbb{R}^{3} \backslash \mathcal{K}\right)} \\
& \quad \leq C(u, T)\left(\left\|v_{0}\right\|_{H_{D}^{1}\left(\mathbb{R}^{3} \backslash \mathcal{K}\right)}+\left\|v_{1}\right\|_{L^{2}\left(\mathbb{R}^{3} \backslash \mathcal{K}\right)}+\int_{0}^{T}\|\square v(s, \cdot)\|_{L^{2}\left(\mathbb{R}^{3} \backslash \mathcal{K}\right)} d s\right),
\end{aligned}
$$

where

$$
C(u, T)=C \times\left(\left\|u_{0}\right\|_{H_{D}^{2}\left(\mathbb{R}^{3} \backslash \mathcal{K}\right)}+\left\|u_{1}\right\|_{H_{D}^{1}\left(\mathbb{R}^{3} \backslash \mathcal{K}\right)}+\sum_{|\alpha| \leq 1} \int_{0}^{T}\left\|D^{\alpha} \square u(s, \cdot)\right\|_{L^{2}\left(\mathbb{R}^{3} \backslash \mathcal{K}\right)} d s\right) .
$$

By (8.1), if $T^{\prime}<(2 C(u, T))^{-2}$, and $\tilde{v}$ denotes the free solution to the Dirichlet-Cauchy problem $\square \tilde{v}=0$ with data $\left(\tilde{v}(0, \cdot), \partial_{t} \tilde{v}(0, \cdot)\right)=\left(v(0, \cdot), \partial_{t} v(0, \cdot)\right) \in H_{D}^{2} \times H_{D}^{1}$, then the Schwarz inequality shows that the map

$$
G \rightarrow Q\left(d u, d\left(\square^{-1} G+\tilde{v}\right)\right)+Q\left(d\left(\square^{-1} G+\tilde{v}\right), d u\right)+F
$$

is a contraction in the norm $\sum_{|\alpha| \leq 1}\left\|D^{\alpha} G\right\|_{L^{2}\left(\left[0, T^{\prime}\right] \times \mathbb{R}^{3} \backslash \mathcal{K}\right)}$. Here, for shorthand, $\square^{-1} G$ denotes the solution of the inhomogeneous wave equation with forcing term $G$ and zero initial data. If $G$ is the unique fixed point of this map, then $v=\square^{-1} G+\tilde{v}$ satisfies $\square v=Q(d u, d v)+Q(d v, d u)+F$. A similar proof using (8.2) shows that there is a unique solution for $0 \leq t \leq T^{\prime}$ among $v$ such that $\square v \in L^{1}\left(\left[0, T^{\prime}\right] ; L^{2}\left(\mathbb{R}^{3} \backslash \mathcal{K}\right)\right)$. By the assumptions of the lemma, these solutions coincide, which establishes the result for $T$ replaced by $T^{\prime}$. By energy estimates, $v\left(T^{\prime}, \cdot\right) \in H_{D}^{2}\left(\mathbb{R}^{3} \backslash \mathcal{K}\right)$, and $\partial_{t} v\left(T^{\prime}, \cdot\right) \in H_{D}^{2}\left(\mathbb{R}^{3} \backslash \mathcal{K}\right)$. The above argument may be repeated to establish the regularity result for $0 \leq t \leq 2 T^{\prime}$, and iteration yields the result for $0 \leq t \leq T$.

Proof of Theorem 1.3. Let $u$ denote the solution given by Theorem 1.1. We shall show that, under the additional assumptions of Theorem 1.3, if $0<T<\infty$ is fixed then $u \in C^{\infty}\left([0, T] \times \mathbb{R}^{3} \backslash \mathcal{K}\right)$. By truncating the data $f, g$ and using finite propagation velocity, we may assume that the data is compactly supported (the constant $\varepsilon_{0}$ is of course independent of the support.) Let $\psi_{j}(x)=\partial_{t}^{j} u(0, x)$ denote the function obtained by formally differentiating $u$ at $t=0$ and expressing the result in terms of $f$ and $g$. By assumption, $\psi_{j} \in C_{0}^{\infty}\left(\mathbb{R}^{3} \backslash \mathcal{K}\right)$, and $\psi_{j}$ vanishes on $\partial \mathcal{K}$. Then, for finite $T, u \in$ $C^{j}\left([0, T] ; H_{D}^{2-j}\left(\mathbb{R}^{3} \backslash \mathcal{K}\right)\right), j=0,1,2$. We differentiate equation (1.3) in $t$ to obtain the following equation for $v=\partial_{t} u$,

$$
\left\{\begin{array}{l}
\square v=Q(d u, d v)+Q(d v, d u), \quad(t, x) \in \mathbb{R}_{+} \times \mathbb{R}^{3} \backslash \mathcal{K} \\
\left.v(t, \cdot)\right|_{\partial \mathcal{K}}=0 \\
v(0, \cdot) \in H_{D}^{2}\left(\mathbb{R}^{3} \backslash \mathcal{K}\right), \partial_{t} v(0, \cdot) \in H_{D}^{1}\left(\mathbb{R}^{3} \backslash \mathcal{K}\right) .
\end{array}\right.
$$

By Lemma 8.1, $v \in C^{j}\left([0, T] ; H_{D}^{2-j}\left(\mathbb{R}^{3} \backslash \mathcal{K}\right)\right), j=0,1,2$, and $D Q(d u, d v), D Q(d v, d u) \in$ $L^{2}\left([0, T] \times \mathbb{R}^{3} \backslash \mathcal{K}\right)$.

Suppose that we have shown $\partial_{t}^{k} u \in C^{j}\left([0, T] ; H_{D}^{2-j}\left(\mathbb{R}^{3} \backslash \mathcal{K}\right)\right), j=0,1,2$, for $0 \leq k<$ $m$. We differentiate (1.3) $m$ times in $t$ to obtain the following equation for $v=\partial_{t}^{m} u$,

$$
\left\{\begin{array}{l}
\square v=Q(d u, d v)+Q(d v, d u)+F, \quad(t, x) \in \mathbb{R}_{+} \times \mathbb{R}^{3} \backslash \mathcal{K} \\
\left.v(t, \cdot)\right|_{\partial \mathcal{K}}=0 \\
v(0, \cdot) \in H_{D}^{2}\left(\mathbb{R}^{3} \backslash \mathcal{K}\right), \partial_{t} v(0, \cdot) \in H_{D}^{1}\left(\mathbb{R}^{3} \backslash \mathcal{K}\right)
\end{array}\right.
$$


where

$$
F=\sum_{0<j<m}\left(\begin{array}{c}
m \\
j
\end{array}\right)\left(Q\left(d \partial_{t}^{j} u, d \partial_{t}^{m-j} u\right)+Q\left(d \partial_{t}^{m-j} u, d \partial_{t}^{j} u\right)\right)
$$

By the induction step, and (8.1) and (8.2), we have that $F, D F \in L^{2}\left([0, T] \times \mathbb{R}^{3} \backslash \mathcal{K}\right)$, and $Q(d u, d v), Q(d v, d u) \in L^{2}\left([0, T] \times \mathbb{R}^{3} \backslash \mathcal{K}\right)$. Consequently, $\partial_{t}^{m} u \in C^{j}\left([0, T] ; H_{D}^{2-j}\left(\mathbb{R}^{3} \backslash \mathcal{K}\right)\right)$, for $j=0,1,2$. It follows that

$$
u \in C^{\infty}\left([0, T] ; H_{D}^{2}\left(\mathbb{R}^{3} \backslash \mathcal{K}\right)\right), \quad Q(d u, d u) \in C^{\infty}\left([0, T] ; H^{1}\left(\mathbb{R}^{3} \backslash \mathcal{K}\right)\right),
$$

where, as before, $H^{k}\left(\mathbb{R}^{3} \backslash \mathcal{K}\right)$ denotes Sobolev space of restrictions of elements of $H^{k}\left(\mathbb{R}^{3}\right)$.

We next obtain spatial regularity for $u$ from the equation (1.3). We have

$$
\left\{\begin{array}{l}
\Delta u=\partial_{t}^{2} u+Q(d u, d u) \in C^{\infty}\left([0, T] ; H^{1}\left(\mathbb{R}^{3} \backslash \mathcal{K}\right)\right) \\
\left.u(t, \cdot)\right|_{\partial \mathcal{K}}=0 .
\end{array}\right.
$$

By elliptic regularity, thus $u \in C^{\infty}\left([0, T] ; H_{D}^{3}\left(\mathbb{R}^{3} \backslash \mathcal{K}\right)\right)$. Since $H^{k}\left(\mathbb{R}^{3} \backslash \mathcal{K}\right)$ is an algebra under pointwise multiplication for $k \geq 2$, it follows that $Q(d u, d u) \in C^{\infty}\left([0, T] ; H^{2}\left(\mathbb{R}^{3} \backslash \mathcal{K}\right)\right)$. A simple induction now establishes that $u \in C^{\infty}\left([0, T] ; H_{D}^{k}\left(\mathbb{R}^{3} \backslash \mathcal{K}\right)\right)$ for all $k$.

Remark. If it is assumed that the initial data $\psi_{j}=\partial_{t}^{j} u(0, \cdot)$ belongs to $H_{D}^{2}\left(\mathbb{R}^{3} \backslash \mathcal{K}\right)$ for all $j$, (no smallness assumption is necessary on the norm, except for $j=0,1$ ), then it is not necessary to truncate the data to apply Lemma 8.1. and we may conclude that the solution of Theorem 1.1 satisfies $u \in C^{\infty}\left([0, T] ; H_{D}^{k}\left(\mathbb{R}^{3} \backslash \mathcal{K}\right)\right)$ for all $k$.

\section{REFERENCES}

[1] D. Christodoulou: Solutions globales des equations de champ de Yang-Mills, C.R. Acad. Sci. Paris, 293, Series A, (1981), 481-506.

[2] D. Christodoulou: Global solutions of nonlinear hyperbolic equations for small initial data, Comm. Pure Appl. Math. 39 (1986), 267-282.

[3] V. Georgiev and P. P. Schirmer: Global existence of low regularity solutions of non-linear wave equations, Math. Z. 219 (1995), 1-19.

[4] P. Godin: Global existence of solutions to some exterior radial quasilinear Cauchy-Dirichlet problems, Amer. J. Math. 117 (1995), 1475-1505.

[5] N. Hayashi: Global existence of small radially symmetric solutions to quadratic nonlinear wave equations in an exterior domain, Manuscripta Math. 81 (1993), 15-39.

[6] N. Hayashi: Global existence of small solutinos to quadratic nonlinear wave equatinos in an exterior domain, J. Funct. Anal. 131 (1995), 302-344.

[7] L. Hörmander: Lectures on nonlinear hyperbolic equations, Springer-Verlag, Berlin, 1977.

[8] F. John: Nonlinear wave equations, formation of singularities, University Lecture Series, American Mathematical Society, 1990.

[9] S. Klainerman: Long time behavior of solutions to nonlinear wave equations, Proceedings of the International Congress of Mathematicians, Warsaw, 1982.

[10] S. Klainerman: The null condition and global existence to nonlinear wave equations, Nonlinear systems of partial differential equations in applied mathematics, Part 1 (Santa Fe, N.M., 1984), 293-326, Lectures in Appl. Math., 23, Amer. Math. Soc., Providence, R.I., 1986.

[11] S. Klainerman and M. Machedon: Space-time estimates for null forms and the local existence theorem, Comm. Pure Appl. Math. 46 (1993), 1221-1268.

[12] P. D. Lax, C. S. Morawetz, and R. S. Phillips: Exponential decay of solutions of the wave equation in the exterior of a star-shaped obstacle, Comm. Pure Appl. Math. 16 (1963), 477-486.

[13] P. D. Lax and S. Phillips: Scattering theory, revised edition, Academic Press, San Diego, 1989.

[14] G. Mockenhaupt, A. Seeger and C. Sogge: Local smoothing of Fourier integral operators and Carleson-Sjölin estimates J. Amer. Math. Soc. 6 (1993), 65-130. 
[15] R. Penrose: Conformal treatment of infinity, 1964 Relativité, Groupes et Topologie , pp. 565-584 Gordon and Breach, New York.

[16] Y. Shibata and Y. Tsutsumi: On a global existence theorem of small amplitude solutions for nonlinear wave equations in an exterior domain, Math. Z. 191 (1986), 165-169.

[17] H. Smith and C. D. Sogge: On the critical semilinear wave equation outside convex obstacles, J. Amer. Math. Soc. 8 (1995), 879-916.

[18] H. Smith and C. D. Sogge: Null form estimates for (1/2,1/2) symbols and local existence for a quasilinear Dirichlet-wave equation, to appear in Ann. Sci. École Norm. Sup.

[19] H. Smith and C. D. Sogge: Global Strichartz estimates for nontrapping perturbations of the Laplacian, to appear in Comm. Partial Differential Equations.

[20] C. D. Sogge: On local existence for nonlinear wave equations satisfying variable coefficient null conditions, Comm. Partial Differential Equations 18 (1993), 1795-1821.

[21] R. Strichartz: A priori estimates for the wave equation and some applications, J. Functional Analysis 5 (1970), 218-235.

Department of Mathematics, California Institute of Technology, Pasadena, CA 91125

Department of Mathematics, University of Washington, Seattle, Wa 98195

Department of Mathematics, The Johns Hopkins University, Baltimore, MD 21218 